\title{
Structure constants of the OSP(1|2) WZNW model
}

\author{
Yasuaki Hikida and Volker Schomerus \\ DESY Theory Group, DESY Hamburg, Notkestrasse 85, D-22603 Hamburg, Germany \\ E-mail: yasuaki.hikida@desy.de, volker.schomerus@desy.de
}

\begin{abstract}
We propose exact formulas for the 2- and 3-point functions of the WZNW model on the non-compact supergroup $\operatorname{OSP}(1 \mid 2)$. Using the path integral approach that was recently developed in arXiv:0706.1030 we show how local correlation functions in the $\operatorname{OSP}(p \mid 2)$ WZNW models can be obtained from those of $\mathcal{N}=p$ supersymmetric Liouville field theory for $p=1,2$. We then employ known results on correlators in $\mathcal{N}=1$ Liouville theory to determine the structure constants of the $\operatorname{OSP}(1 \mid 2)$ theory.
\end{abstract}

Keywords: Conformal and W Symmetry, Conformal Field Models in String Theory. 


\section{Contents}

1. Introduction 1

2. Supergroup models and super Liouville field theory 5

$2.1 \operatorname{OSP}(1 \mid 2)$ WZNW model from $\mathcal{N}=1$ Liouville theory 5

$2.2 \operatorname{OSP}(2 \mid 2)$ WZNW model from $\mathcal{N}=2$ super Liouville theory 9

3. OSP(1|2) WZNW model - the particle limit 12

$\begin{array}{lll}3.1 & \text { Particle wave functions on } \operatorname{OSP}(1 \mid 2) & 12\end{array}$

$\begin{array}{ll}3.2 \text { Wave functions - another basis } & 14\end{array}$

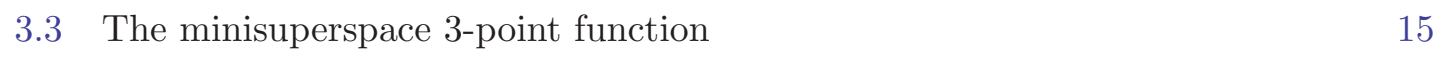

4. Solution of the $\operatorname{OSP}(1 \mid 2)$ WZNW model 17

$\begin{array}{lll}4.1 & \text { The WZNW-Liouville correspondence revisited } & 17\end{array}$

$\begin{array}{lll}4.2 & \text { Computation of 2-point functions } & 18\end{array}$

$\begin{array}{ll}4.3 \text { Computation of 3-point functions } & 18\end{array}$

$\begin{array}{ll}\text { 5. Conclusion } & 21\end{array}$

A. The Lie superalgebras $\operatorname{osp}(1 \mid 2)$ and $\operatorname{sl}(1 \mid 2) \quad 23$

$\begin{array}{lll}\text { A.1 The Lie superalgebra osp }(1 \mid 2) & 23\end{array}$

$\begin{array}{ll}\text { A.2 The Lie superalgebra } \operatorname{osp}(2 \mid 2) & 24\end{array}$

B. Integral formulas $\quad 24$

C. $\mathcal{N}=1$ super Liouville theory $\quad 26$

\section{Introduction}

Two dimensional conformal field theories with target space supersymmetry have recently been studied intensively because of the important role they play for various problems ranging from disordered electron systems to string theory. Through the AdS/CFT correspondence, for instance, 4-dimensional theories with superconformal symmetry are related to closed strings moving in a target space with psl $(2,2 \mid 4)$ symmetry (see e.g. [1] for a concrete world-sheet model). In condensed matter theory, on the other hand, methods have been developed [2] which permit the computation of spectral densities, transport properties, etc., in systems with random disorder. These involve auxiliary field theories with internal supersymmetry, in particular models with central charge $c=0$ whose action is invariant under global $\operatorname{osp}(2 \mathrm{~N} \mid 2 \mathrm{~N})$ transformations (see e.g. [3] for a review and further references). 
Conformal field theories on superspaces provide a rich class of non-unitary and nonrational models. Moreover, their correlators often possess logarithmic singularities, a property that was explained through harmonic analysis on supergroups in [4]. Given all these features, models with target space supersymmetry may appear rather difficult to solve! Some progress was made recently for a large class of basic Lie superalgebras. In fact, the solution of WZNW models on type I supergroups has been reduced to solving an associated bosonic WZNW model. The type I condition (see below for more details) is satisfied for many interesting examples, in particular for the supergroups PSL $(\mathrm{N} \mid \mathrm{N})$, but it excludes almost all supergroups $\operatorname{OSP}(\mathrm{M} \mid 2 \mathrm{~N})$, with the exception of $\operatorname{OSP}(2 \mid 2 \mathrm{~N})$. Therefore, the solution of WZNW models on $\operatorname{OSP}(\mathrm{M} \mid 2 \mathrm{~N})$ remains an important open problem. In this note we shall develop a new approach to the issue and we shall employ it to solve the WZNW model on $\operatorname{OSP}(1 \mid 2)$.

For a moment we shall put the topic of sigma models on superspaces aside and talk about a somewhat unrelated development in the area of non-rational conformal field theory (CFT). The two most thoroughly studied examples of CFTs with non-compact target space are Liouville theory and the WZNW model on the 3-dimensional hyperboloid $\mathrm{H}_{3}^{+}$, a Euclidean version of $A d S_{3}$. The structure constants of Liouville field theory were first proposed by Dorn, Otto and the Zamolodchikovs [5, 6]. The original proposal has meanwhile been established rigorously through a series of papers by Teschner, partly in collaboration with Ponsot $[7,8,9]$. At the same time, Teschner also extended the successful solution of Liouville theory to the $H_{3}^{+}$model $[10,11]$. It turned out that the structure constants of Liouville theory appear as building blocks for those of the WZNW model. This may not come entirely unexpected since it is often stated that Liouville theory provides a model for the radial direction of $A d S$. In any case, the close relation between the two theories has furnished rather useful tools, e.g. to prove consistency of structure functions in the $H_{3}^{+}$model [12]. A precise relation between local correlation functions of the $H_{3}^{+}$WZNW model and Liouville field theory on the complex plane was later put together by Ribault and Teschner [13], based on earlier related works [15, 14].

In our recent paper [16] we revisited and extended the relation between the WZNW model on $\mathrm{H}_{3}^{+}$and Liouville field theory. Most importantly, we rederived the relation found in [13] very naturally using a path integral formalism. As one application of our new approach we then generalized the correspondence to correlation functions on arbitrary closed surfaces. The path integral treatment clearly suggests that similar correspondences may exist for other models with an $\mathrm{sl}_{2}$ current algebra symmetry. Furthermore, whenever this is the case, the path integral approach provides concrete tools to determine the precise relation between local correlation functions of two models. We shall often refer to such a correspondence as a 'reduction' simply because it expresses correlation functions of a model on a $d$-dimensional target space through correlators in another local field theory with target space of dimension $d-2$. Let us stress, however, that the correspondence goes far beyond the well known Hamiltonian reduction (see [17] for a review and references to early contributions). Most importantly, the new correspondence imposes absolutely no restrictions on the momenta of the tachyon vertex operators, in spite of the difference 
between target space dimensions. While target space momenta in the original theory with $\mathrm{sl}_{2}$ symmetry possess $d$ parameters, only $d-2$ components are needed to parametrize momenta in the reduced model. The correspondence stores all information about the missing two momentum components through a highly intriguing mechanism in the worldsheet location of additional degenerate field insertions.

Let us now connect the correspondence between the $H_{3}^{+}$WZNW model and Liouville theory (or its generalizations) to the main goal of this work, namely the solution of WZNW models on OSP supergroups. All classical Lie superalgebras possess a so-called distinguished $\mathbb{Z}$-gradation in which fermionic generators possess degree $\delta= \pm 1$. For type I superalgebras, all bosonic generators are located in degree zero. Because there exist no bosonic elements with degree $\delta=+2$, anti-commutators between fermionic elements of degree $\delta=+1$ vanish. This feature of type I superalgebras was exploited in [18] and leads to a solution of the corresponding WZNW models. For type II superalgebras the situation is different. By definition, these contain bosonic generators of degree $\delta= \pm 2$ with respect to the distinguished $\mathbb{Z}$-gradation. Consequently, anti-commutators between fermionic elements of degree $\delta=+1$ need no longer vanish, a property that spoils a successful solution along the lines of [18]. Our idea here is to remove the problematic generators of degree $\delta= \pm 2$ through reduction to a local field theory with a lower dimensional target space. Note that each generator of degree $\delta=+2$ determines a unique $\mathrm{sl}_{2}$ subalgebra within the current superalgebra of a type II WZNW model. Moreover, the different $\mathrm{sl}_{2}$ current algebras that are obtained in this way commute with each other. Therefore, we can apply the 'reduction' outlined in the previous paragraph to each of the $\mathrm{sl}_{2}$ algebras, one after another. In this note we shall restrict ourselves to examples with a single bosonic generator of degree $\delta=+2$. In fact, most of our analysis focuses even further to the example of osp $(1 \mid 2)$.

Let us now outline the main results of this paper and describe the content of each of the following sections. We shall begin in section 2 by spelling out the action of the $\operatorname{OSP}(1 \mid 2)$ WZNW model in a first order formulation. The Lie superalgebra osp (1|2) contains a bosonic $\mathrm{sl}_{2}$ subalgebra along with two fermionic generators. After 'reduction' there remains a single bosonic direction and two fermionic ones. The latter are shown to provide the fermionic partner of the bosonic field in $\mathcal{N}=1$ Liouville field theory and an additional free fermion. Thereby, we shall relate correlators in the OSP $(1 \mid 2)$ WZNW model to those in a product of $\mathcal{N}=1$ Liouville theory with a free fermion model. We shall then briefly demonstrate how our strategy extends to higher dimensional OSP supergroups. In particular, we relate the $\operatorname{OSP}(2 \mid 2)$ WZNW model to $\mathcal{N}=2$ Liouville theory. ${ }^{1}$ The relation between $\operatorname{osp}(p \mid 2)$ current algebras and $\mathcal{N}=p$ superconformal symmetries is not new. In fact, it was known for a long time how to obtain the latter from the former through Hamiltonian reduction [19]. But let us stress once more that our correspondence goes much beyond a mere reduction since it establishes an equivalence between correlators of the two theories.

\footnotetext{
${ }^{1}$ As we remarked before, the supergroup $\operatorname{OSP}(2 \mid 2) \cong \mathrm{SL}(1 \mid 2)$ is of type I and therefore it can be dealt with along the lines of [18]. Our treatment here shall be based on a natural $\mathbb{Z}$-gradation in which $\operatorname{OSP}(2 \mid 2)$ possesses a single bosonic generator in degrees $\delta= \pm 2$ (i.e. not the distinguished $\mathbb{Z}$-gradation). This makes it an example for our new approach and thereby provides an alternative way of solving the model, different from the one outlined in [18].
} 
The rest of the paper is then devoted to the computation of 2- and 3-point functions in the OSP (1|2) WZNW model. In section 3 we shall study the minisuperspace approximation to the structure constants. This will also allow us to gain some experience with the osp $(1 \mid 2)$ invariant tensors which appear as building blocks for the particle and field theory quantities alike. Section 4 contains formulas for the structure constants of the OSP(1|2) WZNW model. The 2-point functions of the theory can be determined easily from the known 2point functions of $\mathcal{N}=1$ Liouville field theory. The 3-point functions of the WZNW model are related to certain 4-point functions in the reduced model. Luckily, the relevant 4-point correlators in $\mathcal{N}=1$ Liouville theory have been constructed in [20]. When combined with the appropriate correlation functions in free fermionic field theory, the resulting 3-point function of the WZNW model becomes manifestly $\operatorname{osp}(1 \mid 2)$ invariant and the structure constants can be read off.

Our final formula for the 3-point correlator will involve tachyon vertex operators $V^{j}(x, \xi \mid z)=V^{j}(x, \bar{x}, \xi, \bar{\xi} \mid z)$ which are labeled by a spin $j$, a complex coordinate $x$ and a complex Grassmann variable $\xi$. From time to time we shall also display the dependence on the complex conjugate variables $\bar{x}$ and $\bar{\xi}$ in order to show that the corresponding quantities are not chiral. The parameters $j, x, \xi$ are chosen such that the operator products with supercurrents $J_{X}$ of the $\operatorname{OSP}(1 \mid 2)$ WZNW model take the form

$$
J_{X}(z) V^{j}(x, \xi \mid w) \sim r_{X} V^{j}(x, \xi \mid w) \frac{1}{z-w}+\ldots .
$$

Here, the subscript $X$ runs through a basis $X=E^{ \pm}, F^{ \pm}, H$ in osp(1|2) (see appendix A.1 for details on osp(1|2)). The symbols $r_{X}$ on the right hand side of the operator product denote certain first order differential operators acting on $x, \bar{x}$ and $\xi, \bar{\xi}$, see eqs. (3.15). The 3 -point function of tachyon vertex operators reads

$$
\begin{aligned}
\left\langle V^{j_{1}}\left(x_{1}, \xi_{1} \mid z_{1}\right) V^{j_{2}}\right. & \left.\left(x_{2}, \xi_{2} \mid z_{2}\right) V^{j_{3}}\left(x_{3}, \xi_{3} \mid z_{3}\right)\right\rangle \\
& =\frac{C_{b}\left(j_{1}, j_{2}, j_{3}\right)+\tilde{C}_{b}\left(j_{1}, j_{2}, j_{3}\right) \eta \bar{\eta}}{\left|X_{12}\right|^{-2 j_{12}-1}\left|X_{23}\right|^{-2 j_{23}-1}\left|X_{31}\right|^{-2 j_{31}-1}} \frac{1}{\prod_{i<j}\left|z_{i j}\right|^{2 \Delta_{i j}}}
\end{aligned}
$$

where $z_{i j}=z_{i}-z_{j}, j_{12}=j_{1}+j_{2}-j_{3}$ etc. and $X_{i j}=x_{i}-x_{j}-\xi_{i} \xi_{j}$. The exponents $\Delta_{i j}$ are determined by the conformal dimensions

$$
\Delta_{j}=-2 b^{2}(j+1)\left(j+\frac{1}{2}\right) \quad \text { where } b^{-2}=2 k-3
$$

through $\Delta_{12}=\Delta_{j_{1}}+\Delta_{j_{2}}-\Delta_{j_{3}}$ etc. An explicit formula for the super-projective 3-point invariants $\eta, \bar{\eta}$ is given in eq. (3.20). The form of the 3 -point functions is determined by world-sheet conformal symmetry and target space $\operatorname{osp}(1 \mid 2)$ invariance up to the two functions $C_{b}$ and $\tilde{C}_{b}$. Expressions for these are provided in eqs. (4.19) and (4.21) at the very end of this note. Thereby, the non-rational OSP(1|2) WZNW model is solved. Structure constants for a compact target space may be obtained by analytic continuation of the momenta. Such models have been argued to describe the continuum limit of certain superspin chains, see [21] and [22] for the cases of $\operatorname{osp}(1 \mid 2)$ and $\operatorname{osp}(2 \mid 2)$, respectively. An $\operatorname{OSP}(2 \mid 2)$ WZNW model also emerges in the study of $2+1$ dimensional spin-full electrons with random gauge potential, see [23, 24, 25] and further references therein. 


\section{Supergroup models and super Liouville field theory}

The aim of this section is to derive a relation between the $\operatorname{OSP}(p \mid 2), p=1,2$, WZNW model and the product of a supersymmetric Liouville theory with a theory of $p$ free fermions. Let us note that the supergroup $\operatorname{OSP}(p \mid 2)$ has superdimension $\operatorname{sdim} \operatorname{OSP}(p \mid 2)=\left(\frac{1}{2}\left(p^{2}-p\right)+\right.$ 3) $\mid 2 p$. The manipulations to be carried out in the current section work for all $p$. They relate the WZNW model to a new interacting field theory on a target space of superdimension $\left(\frac{1}{2}\left(p^{2}-p\right) p+1\right) \mid p$ and an additional model of $p$ free massless fermions. Two bosonic directions are integrated out explicitly while half of the fermions turn out to decouple. When $p=1,2$, the field content of the interacting sector is that of $\mathcal{N}=1,2$ Liouville field theory and we shall see that the actions also agree. For larger values of $p$, the corresponding lower dimensional model has not been studied before so that the relation is of limited use. For this reason, we shall mostly focus on the case of $p=1$ and then spell out the relation for $p=2$. Larger values of $p$ may be treated in the same way.

\subsection{OSP $(1 \mid 2)$ WZNW model from $\mathcal{N}=1$ Liouville theory}

In this subsection, we focus on the simplest example and derive the relation between correlators of OSP $(1 \mid 2)$ WZNW model and $\mathcal{N}=1$ super Liouville field theory. After a few introductory comments on the action of the OSP $(1 \mid 2)$ WZNW model, we shall pass to a first order formulation involving two additional bosonic auxiliary fields along with two fermionic ones. Following the ideas of [16], we can then integrate out four bosonic fields. The resulting theory contains a single bosonic field and two pairs of chiral fermions. Their action is finally rewritten as a sum of an $\mathcal{N}=1$ Liouville model and a free fermion theory.

For any (super-)group, the action of WZNW model takes the following standard form,

$$
S^{\mathrm{WZNW}}(g)=\frac{k}{4 \pi} \int_{\Sigma} d^{2} z\left\langle g^{-1} \partial g, g^{-1} \bar{\partial} g\right\rangle+\frac{k}{24 \pi} \int_{B}\left\langle g^{-1} d g,\left[g^{-1} d g, g^{-1} d g\right]\right\rangle
$$

where the integrations are over a world-sheet $\Sigma$ and a three dimensional manifold with $\partial B=\Sigma$, respectively. The Lie superalgebra osp(1|2) has superdimension $3 \mid 2$ with bosonic and fermionic generators denoted by $E^{ \pm}, H$ and by $F^{ \pm}$, respectively. Their (anti-)commutation relations may be found in appendix A.1 along with explicit formulas for the metric we use.

We shall adopt a specific parametrization of elements $g \in \operatorname{OSP}(1 \mid 2)$ by splitting them into a product $g=\alpha G \beta$ of three $3 \times 3$ supermatrices which are defined by

$$
\alpha=e^{2 \theta F^{+}}, \quad \beta=e^{2 \bar{\theta} F^{-}} \quad G=\left(\begin{array}{cc}
g_{B} & 0 \\
0 & 1
\end{array}\right), \quad g_{B}=\left(\begin{array}{ll}
1 & \gamma \\
0 & 1
\end{array}\right)\left(\begin{array}{cc}
e^{\phi} & 0 \\
0 & e^{-\phi}
\end{array}\right)\left(\begin{array}{ll}
1 & 0 \\
\bar{\gamma} & 1
\end{array}\right) .
$$

The action of the WZNW model can now be spelled out explicitly in terms of three bosonic fields $\phi, \gamma, \bar{\gamma}$ and two fermionic ones $\theta, \bar{\theta}$. To this end, we decompose the elements $g=\alpha G \beta$ into its three factors and then split the WZNW action (2.1) into several terms with the 
help of the Polyakov-Wiegmann identity,

$$
\begin{aligned}
S^{\mathrm{WZNW}}(\alpha G \beta)= & S^{W Z N W}(G)+\frac{k}{2 \pi} \int d^{2} z\left\langle\alpha^{-1} \bar{\partial} \alpha, \partial G G^{-1}\right\rangle \\
& +\frac{k}{2 \pi} \int d^{2} z\left\langle G^{-1} \bar{\partial} G, \partial \beta \beta^{-1}\right\rangle+\frac{k}{2 \pi} \int d^{2} z\left\langle\alpha^{-1} \bar{\partial} \alpha, G \partial \beta \beta^{-1} G^{-1}\right\rangle .
\end{aligned}
$$

Inserting our parametrization of the factors $\alpha, \beta$ and $G$, we obtain the following formulas for the action of the OSP(1|2) WZNW model

$$
S^{\mathrm{WZNW}}(g)=\frac{k}{2 \pi} \int d^{2} z\left[\partial \phi \bar{\partial} \phi+e^{-2 \phi}(\partial \bar{\gamma}-\bar{\theta} \partial \bar{\theta})(\bar{\partial} \gamma-\theta \bar{\partial} \theta)+2 e^{-\phi} \bar{\partial} \theta \partial \bar{\theta}\right] .
$$

Note that the theory is interacting with terms up to forth order in the fermionic fields. For WZNW models on type I supergroups a special parametrization could be found [18] in which the interaction terms are at most quadratic in the fermionic fields. It is a basic feature of the type II case that such a simplification cannot be achieved.

In order to apply the method of [16], it is essential to change the action in the first order formulation. Introducing new bosonic variables $\beta, \bar{\beta}$ as well as the fermionic ones $p, \bar{p}$, all four of weight $\Delta=1$, the action may be rewritten as

$$
\begin{aligned}
S^{\mathrm{WZNW}}(g)=\frac{1}{2 \pi} \int d^{2} z\left[\frac{1}{2} \partial \phi \bar{\partial} \phi\right. & +\frac{b}{8} \sqrt{g} R \phi+\beta(\bar{\partial} \gamma-\theta \bar{\partial} \theta)+\bar{\beta}(\partial \bar{\gamma}-\bar{\theta} \partial \bar{\theta}) \\
& \left.+p \bar{\partial} \theta+\bar{p} \partial \bar{\theta}-\frac{1}{k} \beta \bar{\beta} e^{2 b \phi}-\frac{1}{2 k} p \bar{p} e^{b \phi}\right]
\end{aligned}
$$

with $b=1 / \sqrt{2 k-3}$. Before we continue studying this theory let us briefly convince ourselves that the original model agrees with the first order formulation we propose. The equations of motion for new auxiliary fields read

$$
\begin{array}{ll}
\beta=k e^{-2 b \phi}(\partial \bar{\gamma}-\bar{\theta} \partial \bar{\theta}), & \bar{\beta}=k e^{-2 b \phi}(\bar{\partial} \gamma-\theta \bar{\partial} \theta), \\
p=-2 k e^{-b \phi} \partial \bar{\theta}, & \bar{p}=2 k e^{-b \phi} \bar{\partial} \theta .
\end{array}
$$

Inserting these expressions into the action (2.4) we reproduce the original action (2.3) apart from the additional linear dilaton term that appears in eq. (2.4). The latter arises from the Jacobian in the change of variables. Utilizing the formulas

$$
\ln \operatorname{det}(A \partial B \bar{\partial})=\frac{1}{48 \pi} \int d^{2} z\left(|\partial \ln A|^{2}+|\partial \ln B|^{2}-4 \partial \ln A \bar{\partial} \ln B\right)
$$

and $\sqrt{g} R=-4 \partial \bar{\partial} \ln |\rho|^{2}$ for the world-sheet metric $d s^{2}=|\rho|^{2} d z d \bar{z}$, we obtain the following bosonic contribution

$$
-\ln \operatorname{det}\left(|\rho|^{-2} e^{2 \phi} \partial e^{-2 \phi} \bar{\partial}\right)=-\frac{1}{\pi} \int d^{2} \partial \phi \bar{\partial} \phi+\frac{1}{8 \pi} \int d^{2} \phi \sqrt{g} R \phi,
$$

along with a fermionic contribution of the same form

$$
\ln \operatorname{det}\left(|\rho|^{-2} e^{\phi} \partial e^{-\phi} \bar{\partial}\right)=\frac{1}{4 \pi} \int d^{2} \partial \phi \bar{\partial} \phi-\frac{1}{16 \pi} \int d^{2} \phi \sqrt{g} R \phi .
$$


This concludes our derivation of the action (2.4) from the generic formulation (2.1) of the WZNW model on the supergroup $\operatorname{OSP}(1 \mid 2)$.

We are now prepared to begin analyzing correlation functions in the OSP $(1 \mid 2)$ WZNW model. The $N$-point functions of tachyon vertex operators are given by

$$
\left\langle\prod_{\nu=1}^{N} V_{j_{\nu}}^{\epsilon_{\nu}, \bar{\epsilon}_{\nu}}\left(\mu_{\nu} \mid z_{\nu}\right)\right\rangle=\int \mathcal{D} \phi \mathcal{D}^{2} \beta \mathcal{D}^{2} \gamma \mathcal{D}^{2} \theta \mathcal{D}^{2} p e^{-S^{\mathrm{WZNW}}(g)} \prod_{\nu=1}^{N} V_{j_{\nu}}^{\epsilon_{\nu}, \bar{\epsilon}_{\nu}}\left(\mu_{\nu} \mid z_{\nu}\right) .
$$

The vertex operators $V_{j}^{\epsilon, \bar{\epsilon}}(\mu \mid z)$ we inserted in the points $z=z_{\nu}$ depend on the $\operatorname{SL}(2, \mathbb{C})$ quantum numbers $j, \mu, \bar{\mu}$ and an additional choice of $\epsilon, \bar{\epsilon}= \pm 1$. They are defined by

$$
V_{j}^{\epsilon, \bar{\epsilon}}(\mu \mid z)=e^{\epsilon \sqrt{\mu} \theta} e^{\bar{\epsilon} \sqrt{\mu} \bar{\theta}}|\mu|^{2 j+2} e^{\mu \gamma-\bar{\mu} \bar{\gamma}} e^{2 b(j+1) \phi} .
$$

The bosonic factor is the same as in [16]. As one may see by expanding exponentials, the fermionic factors are sufficient to generate $1, \theta, \bar{\theta}$ and $\theta \bar{\theta}$. The basis we have chosen, including the factors $\sqrt{\mu}$ and $\sqrt{\mu}$ in front of the fermionic fields, turn out to be very convenient for what we are about to do.

Having set up our problem, we proceed along the lines of [16] and integrate out $\gamma, \bar{\gamma}$ first and then $\beta, \bar{\beta}$ using the following change of variables

$$
\sum_{\nu=1}^{N} \frac{\mu_{\nu}}{w-z_{\nu}}=u \frac{\prod_{i=1}^{N-2}\left(w-y_{i}\right)}{\prod_{\nu=1}^{N}\left(w-z_{\nu}\right)}=: u \mathcal{B}\left(y_{i}, z_{\nu} ; w\right)
$$

and a similar equation for the conjugate variables. This relation defines the parameter $u$ and the world-sheet coordinates $y_{i}$ in terms of $\mu_{\nu}$. After an appropriate redefinition of the scalar field $\phi$ (see [16] for many more details) we obtain

$$
\begin{aligned}
\left\langle\prod_{\nu=1}^{N} V_{j_{\nu}}^{\epsilon_{\nu}, \bar{\epsilon}_{\nu}}\left(\mu_{\nu} \mid z_{\nu}\right)\right\rangle & =\delta^{2}\left(\sum_{\nu=1}^{N} \mu_{\nu}\right)|u|\left|\tilde{\Theta}_{N}\right|^{2} \int \mathcal{D} \varphi \mathcal{D}^{2} \theta \mathcal{D}^{2} p e^{-S[\varphi, \theta, p]} \times \\
& \times \prod_{\nu=1}^{N} e^{\epsilon_{\nu} \sqrt{\mu_{\nu}} \theta+\bar{\epsilon}_{\nu} \sqrt{\mu_{\nu}} \bar{\theta}} e^{2\left(b\left(j_{\nu}+1\right)+\frac{1}{4 b}\right) \varphi}\left(z_{\nu}\right) \prod_{j=1}^{N-2} e^{-\frac{1}{2 b} \varphi}\left(y_{j}\right),
\end{aligned}
$$

where the new action is now given by

$$
\begin{aligned}
S[\varphi, \theta, p]=\frac{1}{2 \pi} \int d^{2} z\left[\frac{1}{2} \partial \varphi \bar{\partial} \varphi\right. & +\frac{Q_{\varphi}}{8} \sqrt{g} R \varphi-u \mathcal{B} \theta \bar{\partial} \theta+\bar{u} \overline{\mathcal{B}} \bar{\theta} \partial \bar{\theta} \\
& \left.+p \bar{\partial} \theta+\bar{p} \partial \bar{\theta}+\frac{1}{k} e^{2 b \varphi}+\frac{i}{2 k} \frac{1}{|u \mathcal{B}|} p \bar{p} e^{b \varphi}\right] .
\end{aligned}
$$

The background charge for the new scalar field $\varphi$ is shifted from $Q_{\phi}=b$ to $Q_{\varphi}=b+1 / b$ and we also introduced the shorthand

$$
\tilde{\Theta}_{N}=\prod_{\mu<\nu}^{N}\left(z_{\mu}-z_{\nu}\right)^{\frac{1}{4 b^{2}}} \prod_{i<j}^{N-2}\left(y_{i}-y_{j}\right)^{\frac{1}{4 b^{2}}} \prod_{\nu=1}^{N} \prod_{i=1}^{N-2}\left(z_{\nu}-y_{i}\right)^{-\frac{1}{4 b^{2}}} .
$$


As it stands, the action still includes an explicit dependence on the world-sheet coordinates $z_{\nu}$ and $y_{i}$ through the function $\mathcal{B}$ that we introduced in eq. (2.12).

Our next step is to absorb the unwanted factors $\mathcal{B}$ through a redefinition of the fermionic fields. In an intermediate step, we introduce

$$
p^{\prime}:=p / \sqrt{u \mathcal{B}}, \quad \bar{p}^{\prime}:=\bar{p} / \sqrt{-\bar{u} \overline{\mathcal{B}}}, \quad \theta^{\prime}:=\theta \sqrt{u \mathcal{B}}, \quad \bar{\theta}^{\prime}:=\bar{\theta} \sqrt{-\bar{u} \overline{\mathcal{B}}} .
$$

When rewritten through the new fermionic variables, the kinetic terms become

$$
-u \mathcal{B} \theta \bar{\partial} \theta+p \bar{\partial} \theta=-\theta^{\prime} \bar{\partial} \theta^{\prime}+p^{\prime} \bar{\partial} \theta^{\prime}-\left(p^{\prime} \theta^{\prime}+\frac{1}{2} \partial \ln \sqrt{u \mathcal{B}}\right) \bar{\partial} \ln \sqrt{u \mathcal{B}}
$$

Here, the non-trivial shift from $p^{\prime} \theta^{\prime}$ to $p^{\prime} \theta^{\prime}+\frac{1}{2} \partial \ln \sqrt{u \mathcal{B}}$ is a result of regularization. At the same time, the fermionic terms $\exp \left(\epsilon \sqrt{\mu} \theta\left(z_{\nu}\right)\right)$ get replaced by $\exp \left(\epsilon \theta^{\prime}\left(z_{\nu}\right)\right){ }^{2}$ Note that the function $\sqrt{u \mathcal{B}}$ has weight $\Delta=1 / 2$ so that after the redefinition, our new fermionic fields $\theta^{\prime}, \bar{\theta}^{\prime}, p^{\prime}, \bar{p}^{\prime}$ all possess the same weight $\Delta=1 / 2$. We can make the kinetic terms look more symmetric if we adopt the following new basis for fermions,

$$
\chi:=\frac{i}{\sqrt{2}}\left(2 \theta^{\prime}-p^{\prime}\right), \quad \psi:=\frac{1}{\sqrt{2}} p^{\prime}, \quad \bar{\chi}:=-\frac{i}{\sqrt{2}}\left(2 \bar{\theta}^{\prime}-\bar{p}^{\prime}\right), \quad \bar{\psi}:=\frac{1}{\sqrt{2}} \bar{p}^{\prime} .
$$

After inserting these expressions, the chiral kinetic terms read

$$
-u \mathcal{B} \theta \bar{\partial} \theta+p \bar{\partial} \theta=\frac{1}{2} \chi \bar{\partial} \chi+\frac{1}{2} \psi \bar{\partial} \psi+\left(i \psi \chi-\frac{1}{2} \partial \ln \sqrt{u \mathcal{B}}\right) \bar{\partial} \ln \sqrt{u \mathcal{B}} .
$$

The fermionic contribution $\exp \left(\epsilon \theta^{\prime}\left(z_{\nu}\right)\right)=1+\epsilon \theta^{\prime}\left(z_{\nu}\right)$ gets replaced by $1+\epsilon(\psi-i \chi) / \sqrt{2}$. Note that both the vertex operators and the term involving $\psi \chi$ in the action mix the two fermions. In addition, the action still contains terms with $z_{\nu}$-dependent coefficients. In order to proceed, we now bosonize the two fermionic fields $\psi$ and $\chi$,

$$
\psi \pm i \chi=\sqrt{2} \exp ( \pm i Y), \quad \bar{\psi} \pm i \bar{\chi}= \pm i \sqrt{2} \exp ( \pm i \bar{Y}) .
$$

The main advantage of this bosonization is that we can now express the product $\psi \chi$ as a derivative. Thereby, we may now rewrite the $z_{\nu}$-dependent terms in the action as follows,

$$
\begin{aligned}
& i \psi(w) \chi(w) \bar{\partial} \ln \sqrt{u \mathcal{B}}=-i \partial Y(w) \bar{\partial} \ln \sqrt{u \mathcal{B}} \\
& \sim i Y(w) \partial \bar{\partial} \ln \sqrt{u \mathcal{B}}=-\pi i Y(w)\left[\sum_{\nu=1}^{N} \delta^{2}\left(w-z_{\nu}\right)-\sum_{i=1}^{N-2} \delta^{2}\left(w-y_{i}\right)\right]
\end{aligned}
$$

The symbol $\sim$ means equality up to total derivatives. In the new form, we recognize that the corresponding terms only lead to contributions which are localized at the points $z_{\nu}$

\footnotetext{
${ }^{2}$ We use a regularization scheme $\lim _{w \rightarrow z}(w-z)=-\ln \rho(z)$ for the world-sheet metric $d s^{2}=|\rho(z)|^{2} d z d \bar{z}$. For more details, see [16].
} 
and $y_{i}$ of the world-sheet, i.e. they modify the vertex operators. Our final result for the correlation function of tachyon vertex operators in the OSP $(1 \mid 2)$ WZNW model now reads

$$
\begin{aligned}
& \left\langle\prod_{\nu=1}^{N} V_{j_{\nu}}^{\epsilon_{\nu}, \bar{\epsilon}_{\nu}}\left(\mu_{\nu} \mid z_{\nu}\right)\right\rangle=\delta^{2}\left(\sum_{\nu=1}^{N} \mu_{\nu}\right)\left|\Theta_{N}^{(1)}\right|^{2} \int \mathcal{D} \varphi \mathcal{D}^{2} \psi \mathcal{D}^{2} \chi e^{-S[\varphi, \psi, \chi]} \times \\
& \quad \times \prod_{\nu=1}^{N}\left(e^{\frac{i}{2} Y}+\epsilon e^{-\frac{i}{2} Y}\right)\left(e^{-\frac{i}{2} \bar{Y}}-\bar{\epsilon} e^{\frac{i}{2} \bar{Y}}\right) e^{2\left(b\left(j_{\nu}+1\right)+\frac{1}{4 b}\right) \varphi}\left(z_{\nu}\right) \prod_{j=1}^{N-2} e^{-\frac{i}{2}(Y-\bar{Y})} e^{-\frac{1}{2 b} \varphi}\left(y_{j}\right) .
\end{aligned}
$$

The factor $\Theta_{N}^{(1)}$ combines the function $\tilde{\Theta}_{N}$ we had defined previously in eq. (2.15) with the numerical contributions from the second term in eq. (2.18),

$$
\Theta_{N}^{(1)}=u \prod_{\mu<\nu}^{N}\left(z_{\mu}-z_{\nu}\right)^{\frac{1}{4 b^{2}}-\frac{1}{4}} \prod_{i<j}^{N-2}\left(y_{i}-y_{j}\right)^{\frac{1}{4 b^{2}}-\frac{1}{4}} \prod_{\nu=1}^{N} \prod_{i=1}^{N-2}\left(z_{\nu}-y_{i}\right)^{-\frac{1}{4 b^{2}}+\frac{1}{4}} .
$$

After all our manipulations, the functional $S=S(\varphi, \psi, \chi)$ is a sum of the action for $\mathcal{N}=1$ super Liouville theory $(\varphi, \psi, \bar{\psi})$ and the action for a non-interacting real massless fermion $(\chi, \bar{\chi})$, i.e.

$$
\begin{aligned}
S[\varphi, \psi, \chi]=\frac{1}{4 \pi} \int d^{2} z & {\left[\partial \varphi \bar{\partial} \varphi+\frac{Q_{\varphi}}{4} \sqrt{g} R \varphi+\frac{2}{k} e^{2 b \varphi}+\right.} \\
& \left.+\psi \bar{\partial} \psi+\bar{\psi} \partial \bar{\psi}+\chi \bar{\partial} \chi+\bar{\chi} \partial \bar{\chi}-\frac{2}{k} \psi \bar{\psi} e^{b \varphi}\right] .
\end{aligned}
$$

The vertex operators we insert at $z_{\nu}$ and $y_{i}$ have been written in terms of chiral components $Y$ and $\bar{Y}$ of a bosonic field. Through the relations (2.19), we may think of $Y$ and $\bar{Y}$ as local functional on the space of fermionic fields $\psi, \chi$ and $\bar{\psi}, \bar{\chi}$. Later, we shall re-express the relevant exponentials through spin-fields in the fermionic sector.

\subsection{OSP $(2 \mid 2)$ WZNW model from $\mathcal{N}=2$ super Liouville theory}

In the previous subsection, we have shown that the correlators of $\operatorname{OSP}(1 \mid 2)$ model can be expressed in terms of $\mathcal{N}=1$ super Liouville field theory with additional fermions. As we have remarked in the introduction, the ideas work much more generally. As an example of such generalizations, we shall briefly analyze the $\operatorname{OSP}(2 \mid 2)$ model. Even though $\operatorname{OSP}(2 \mid 2)$ $\cong \mathrm{SL}(1 \mid 2)$ is of type I, we shall treat it in the same way as in the $\operatorname{OSP}(1 \mid 2)$ model in the previous section. This amounts to choosing an unusual $\mathbb{Z}$-grading in which the four fermions are assigned grades $\delta= \pm 1$ such that there exists one bosonic generator of grade $\delta= \pm 2$ each. For the readers' convenience we have listed the anti-commutation relations of the Lie superalgebra osp $(2 \mid 2)$ in appendix A.2.

In order to spell out the action of the WZNW model we need to adopt specific coordinates on supergroup $\operatorname{OSP}(2 \mid 2)$. Here we shall use the parametrization $g=\alpha G \beta$ with

$$
\alpha=e^{\theta_{1} F^{+}+\theta_{2} \bar{F}^{+}}, \quad \beta=e^{\bar{\theta}_{1} F^{-}+\bar{\theta}_{2} \bar{F}^{-}}, \quad G=\left(\begin{array}{cc}
g_{B} & 0 \\
0 & e^{2 i \varphi_{1}}
\end{array}\right),
$$


where the bosonic part is

$$
g_{B}=e^{i \varphi_{1}}\left(\begin{array}{ll}
1 & \gamma \\
0 & 1
\end{array}\right)\left(\begin{array}{cc}
e^{\phi_{2}} & 0 \\
0 & e^{-\phi_{2}}
\end{array}\right)\left(\begin{array}{ll}
1 & 0 \\
\bar{\gamma} & 1
\end{array}\right)
$$

All our notations and conventions regarding $\operatorname{osp}(2 \mid 2)$ may be found in appendix A.2. In this parametrization, the WZNW action becomes

$$
\begin{aligned}
S^{\mathrm{WZNW}}(g) & =\frac{k}{2 \pi} \int d^{2} z\left[\partial \varphi_{1} \partial \varphi_{1}+\partial \phi_{2} \bar{\partial} \phi_{2}+e^{-i \varphi_{1}-\phi_{2}} \bar{\partial} \theta_{1} \partial \bar{\theta}_{2}+e^{i \varphi_{1}-\phi_{2}} \bar{\partial} \theta_{2} \partial \bar{\theta}_{1}\right] \\
& +\frac{k}{2 \pi} \int d^{2} z e^{-2 \phi_{2}}\left(\partial \bar{\gamma}+\frac{1}{2}\left(\bar{\theta}_{1} \partial \bar{\theta}_{2}+\bar{\theta}_{2} \partial \bar{\theta}_{1}\right)\right)\left(\bar{\partial} \gamma-\frac{1}{2}\left(\theta_{1} \bar{\partial} \theta_{2}+\theta_{2} \bar{\partial} \theta_{1}\right)\right) .
\end{aligned}
$$

Introducing new variables as before, the action is rewritten as

$$
\begin{aligned}
S^{\mathrm{WZNW}}(g) & =\frac{1}{2 \pi} \int d^{2} z\left[\partial \varphi_{1} \bar{\partial} \varphi_{1}+\partial \phi_{2} \bar{\partial} \phi_{2}\right. \\
& +\beta\left(\bar{\partial} \gamma-\frac{1}{2}\left(\theta_{1} \bar{\partial} \theta_{2}+\theta_{2} \bar{\partial} \theta_{1}\right)\right)+\bar{\beta}\left(\partial \bar{\gamma}+\frac{1}{2}\left(\bar{\theta}_{1} \partial \bar{\theta}_{2}+\bar{\theta}_{2} \partial \bar{\theta}_{1}\right)\right)-\frac{1}{k} \beta \bar{\beta} e^{2 b \phi} \\
& \left.+p_{1} \bar{\partial} \theta_{1}+\bar{p}_{1} \partial \bar{\theta}_{1}+p_{2} \bar{\partial} \theta_{2}+\bar{p}_{2} \partial \bar{\theta}_{2}-\frac{1}{k} p_{1} \bar{p}_{2} e^{b\left(i \varphi_{1}+\phi_{2}\right)}-\frac{1}{k} p_{2} \bar{p}_{1} e^{b\left(-i \varphi_{1}+\phi_{2}\right)}\right]
\end{aligned}
$$

with the parameter $b$ being related to the level $k$ of the WZNW model through $b=$ $1 / \sqrt{k-1}$. Note that this relation differs from the one we found in the case of osp $(1 \mid 2)$.

In the first order formulation the $N$-point function of tachyon vertex operators becomes

$$
\left\langle\prod_{\nu=1}^{N} V_{j_{\nu}}\left(\mu_{\nu} \mid z_{\nu}\right)\right\rangle=\int \mathcal{D} \varphi_{1} \mathcal{D} \phi_{2} \mathcal{D}^{2} \beta \mathcal{D}^{2} \gamma \prod_{a=1}^{2} \mathcal{D}^{2} \theta_{a} \mathcal{D}^{2} p_{a} e^{-S^{\mathrm{WZNW}}[g]} \prod_{\nu=1}^{N} V_{j_{\nu}}\left(\mu_{\nu} \mid z_{\nu}\right)
$$

In our discussion of the $\operatorname{OSP}(2 \mid 2)$ WZNW model we shall restrict ourselves to correlation functions of purely bosonic vertex operators,

$$
V_{j_{\nu}}\left(\mu_{\nu} \mid z_{\nu}\right)=\left|\mu_{\nu}\right|^{2 j_{\nu}+2} e^{\mu_{\nu} \gamma-\bar{\mu}_{\nu} \bar{\gamma}} e^{2 b\left(j_{\nu}+1\right) \phi}
$$

It is not too difficult to work with the full vertex operators, including exponentials of the fermionic fields. Since we have seen above how such terms are taken into account, there is no need to repeat all this for $\operatorname{osp}(2 \mid 2)$ now. Following [16] we integrate out $\beta, \gamma$ to obtain

$$
\begin{aligned}
\left\langle\prod_{\nu=1}^{N} V_{\nu}\left(z_{\nu}\right)\right\rangle & =\delta^{2}\left(\sum_{\nu=1}^{N} \mu_{\nu}\right)\left|\tilde{\Theta}_{N}\right|^{4} \prod_{a=1}^{2} \int \mathcal{D} \varphi_{a} \mathcal{D}^{2} \theta_{a} \mathcal{D}^{2} p_{a} \times \\
& \times e^{-S\left[\varphi_{a}, \theta_{a}, p_{a}\right]} \prod_{\nu=1}^{N} e^{2\left(b\left(j_{\nu}+1\right)+\frac{1}{2 b}\right) \varphi_{2}}\left(z_{\nu}\right) \prod_{j=1}^{N-2} e^{-\frac{1}{b} \varphi_{2}}\left(y_{j}\right),
\end{aligned}
$$


where the action is

$$
\begin{aligned}
S\left[\varphi_{a}, \theta_{a}, p_{a}\right] & =\frac{1}{2 \pi} \int d^{2} z\left[\partial \varphi_{1} \bar{\partial} \varphi_{1}+\partial \varphi_{2} \bar{\partial} \varphi_{2}+\frac{Q_{2}}{4} \sqrt{g} R \varphi_{2}\right. \\
& -\frac{1}{2} u \mathcal{B}\left(\theta_{1} \bar{\partial} \theta_{2}+\theta_{2} \bar{\partial} \theta_{1}\right)-\frac{1}{2} \bar{u} \overline{\mathcal{B}}\left(\bar{\theta}_{1} \partial \bar{\theta}_{2}+\bar{\theta}_{2} \partial \bar{\theta}_{1}\right)+\frac{1}{k} e^{2 b \varphi_{2}} \\
& \left.+p_{1} \bar{\partial} \theta_{1}+\bar{p}_{1} \partial \bar{\theta}_{1}+p_{2} \bar{\partial} \theta_{2}+\bar{p}_{2} \partial \bar{\theta}_{2}+\frac{i}{k} \frac{1}{|u \mathcal{B}|}\left(p_{1} \bar{p}_{2} e^{b\left(i \varphi_{1}+\varphi_{2}\right)}+p_{2} \bar{p}_{1} e^{b\left(-i \varphi_{1}+\varphi_{2}\right)}\right)\right]
\end{aligned}
$$

with $Q_{2}=1 / b$. The factor $\tilde{\Theta}_{N}$ has been introduced previously in eq. (2.15). Now we follow the same steps as in the case of osp(1|2), i.e. we rescale and rotate all the fermionic fields,

$$
\chi_{1}:=i\left(\theta_{1} \sqrt{u \mathcal{B}}-p_{2} / \sqrt{u \mathcal{B}}\right), \quad \chi_{2}:=i\left(\theta_{2} \sqrt{u \mathcal{B}}-p_{1} / \sqrt{u \mathcal{B}}\right), \quad \psi_{a}:=p_{a} / \sqrt{u \mathcal{B}} .
$$

Once more, it is not difficult to rewrite the action in terms of the new fermionic fields in terms of $\chi_{a}, \psi_{a}$. For the kinetic terms this is done with the help of the identity,

$$
\begin{aligned}
& -\frac{1}{2} u \mathcal{B}\left(\theta_{1} \bar{\partial} \theta_{2}+\theta_{2} \bar{\partial} \theta_{1}\right)+\sum_{a} p_{a} \bar{\partial} \theta_{a} \\
& =\chi_{1} \bar{\partial} \chi_{2}+\psi_{1} \bar{\partial} \psi_{2}+\sum_{a}\left(i \psi_{a} \chi_{a}-\frac{1}{2} \partial \ln \sqrt{u \mathcal{B}}\right) \bar{\partial} \ln \sqrt{u \mathcal{B}}
\end{aligned}
$$

In order to spell out the vertex operators, we bosonize all four fermionic fields,

$$
\psi_{a} \pm i \chi_{a}=2 \exp \left(i Y_{a}\right)
$$

Putting all this together, we arrive at the following formula for correlation functions in the WZNW model,

$$
\begin{aligned}
\left\langle\prod_{\nu=1}^{N} V_{j_{\nu}}\left(\mu_{\nu} \mid z_{\nu}\right)\right\rangle & =\delta^{2}\left(\sum_{\nu=1}^{N} \mu_{\nu}\right)\left|\Theta_{N}^{(2)}\right|^{2} \prod_{a=1}^{2} \int \mathcal{D} \varphi_{a} \mathcal{D}^{2} \psi_{a} \mathcal{D}^{2} \chi_{a} e^{-S\left[\phi_{a}, \psi_{a}, \chi_{a}\right]_{X}} \\
& \times \prod_{\nu=1}^{N} e^{\frac{i}{2} \sum_{a}\left(Y_{a}+\bar{Y}_{a}\right)+2\left(b\left(j_{\nu}+1\right)+\frac{1}{2 b}\right) \varphi_{2}}\left(z_{\nu}\right) \prod_{j=1}^{N-2} e^{-\frac{i}{2} \sum_{a}\left(Y_{a}+\bar{Y}_{a}\right)-\frac{1}{b} \varphi_{2}}\left(y_{j}\right),
\end{aligned}
$$

where the action $S$ is built from an $\mathcal{N}=2$ supersymmetric Liouville theory for the fields $\left(\varphi_{a}, \psi_{a}, \bar{\psi}_{a}\right)$ and the theory of two free fermions $\left(\chi_{a}, \bar{\chi}_{a}\right)$,

$$
\begin{aligned}
S[\phi, \psi, \chi] & =\frac{1}{2 \pi} \int d^{2} z\left[\partial \varphi_{1} \bar{\partial} \varphi_{1}+\partial \varphi_{2} \bar{\partial} \varphi_{2}+\frac{Q_{2}}{4} \sqrt{g} R \varphi_{2}+\frac{1}{k} e^{2 b \varphi_{2}}\right. \\
& \left.+\psi_{1} \bar{\partial} \psi_{2}+\bar{\psi}_{1} \partial \bar{\psi}_{2}+\chi_{1} \bar{\partial} \chi_{2}+\bar{\chi}_{1} \partial \bar{\chi}_{2}+\frac{i}{k}\left(\psi_{1} \bar{\psi}_{2} e^{b\left(i \varphi_{1}+\varphi_{2}\right)}+\psi_{2} \bar{\psi}_{1} e^{b\left(-i \varphi_{1}+\varphi_{2}\right)}\right)\right]
\end{aligned}
$$

The factor $\Theta_{N}^{(2)}$ is very similar to the corresponding function in the $\operatorname{OSP}(1 \mid 2)$ model,

$$
\Theta_{N}^{(2)}=u \prod_{\mu<\nu}^{N}\left(z_{\mu}-z_{\nu}\right)^{\frac{1}{2 b^{2}}-\frac{1}{2}} \prod_{i<j}^{N-2}\left(y_{i}-y_{j}\right)^{\frac{1}{2 b^{2}}-\frac{1}{2}} \prod_{\nu=1}^{N} \prod_{i=1}^{N-2}\left(z_{\nu}-y_{i}\right)^{-\frac{1}{2 b^{2}}+\frac{1}{2}}
$$


In conclusion, we have shown that correlators of tachyon vertex operators in the $\operatorname{OSP}(2 \mid 2)$ WZNW model may be obtained from correlation functions in $\mathcal{N}=2$ Liouville field theory. Since we do not know much about the latter when $\mathcal{N} \geq 3$, we shall not exploit this relation any further. Instead, we shall now focus on the OSP(1|2) WZNW model.

\section{OSP(1|2) WZNW model - the particle limit}

Our main aim below is to exploit our relation (2.21) between the OSP(1|2) WZNW model and $\mathcal{N}=1$ Liouville theory along with known results for the latter in order to solve the former. Before going into the full and cumbersome conformal field theory computations, however, it is instructive to examine the particle limit. We shall determine the minisuperspace wave functions in the first subsection and then calculate particle analogues of the 2-point and 3-point functions.

\subsection{Particle wave functions on $\operatorname{OSP}(1 \mid 2)$}

Let us first construct the wave functions for a particle that moves freely on the supergroup $\operatorname{OSP}(1 \mid 2)$. The mathematical problem that needs to be solved is to determine all eigenfunctions of Laplace operator. In our parametrization (2.24), the generators of infinitesimal right translations on $\operatorname{OSP}(1 \mid 2)$ are easily worked out,

$$
\begin{aligned}
& R_{E^{+}}=\partial_{\gamma}, \quad R_{H}=-\frac{1}{2} \partial_{\phi}-\gamma \partial_{\gamma}-\frac{1}{2} \theta \partial_{\theta}, \\
& R_{E^{-}}=e^{2 \phi} \partial_{\bar{\gamma}}-\gamma^{2} \partial_{\gamma}-\gamma \partial_{\phi}-\gamma \theta \partial_{\theta}+e^{\phi} \theta\left(\partial_{\bar{\theta}}-\bar{\theta} \partial_{\bar{\gamma}}\right), \\
& R_{F^{+}}=\frac{1}{2}\left(\partial_{\theta}+\theta \partial_{\gamma}\right), \quad R_{F^{-}}=\frac{1}{2} e^{\phi}\left(\partial_{\bar{\theta}}-\bar{\theta} \partial_{\bar{\gamma}}\right)-\frac{1}{2} \gamma\left(\partial_{\theta}+\theta \partial_{\gamma}\right)-\frac{1}{2} \theta \partial_{\phi} .
\end{aligned}
$$

We may now insert these expressions into the general formula for the quadratic Casimir element and thereby derive the following Laplacian on $\operatorname{OSP}(1 \mid 2)$

$$
\begin{aligned}
\Delta & =H H+\frac{1}{2}\left(E^{+} E^{-}+E^{-} E^{+}\right)-\left(F^{+} F^{-}-F^{-} F^{+}\right) \\
& =\frac{1}{4} \partial_{\phi}^{2}-\frac{1}{4} \partial_{\phi}+e^{2 \phi} \partial_{\gamma} \partial_{\bar{\gamma}}-\frac{1}{2} e^{\phi}\left(\partial_{\theta}-\theta \partial_{\gamma}\right)\left(\partial_{\bar{\theta}}-\bar{\theta} \partial_{\bar{\gamma}}\right) .
\end{aligned}
$$

The construction of eigenfunction proceeds in several steps. To begin with, we shall look for eigenfunctions $\Phi^{j}$ of the following special form

$$
\Phi^{j}(\gamma, \bar{\gamma}, \theta, \bar{\theta}, \phi)=e^{\mu \gamma-\bar{\mu} \bar{\gamma}} \phi_{\mu \bar{\mu}}^{j}(\theta, \bar{\theta}, \phi) .
$$

Here, $j$ parametrizes the eigenvalue $\lambda=(j+1)\left(j+\frac{1}{2}\right)$ of the Laplacian. With our ansatz, we have explicitly diagonalized the operators $R_{E^{+}}$and $L_{E^{-}}=\partial_{\bar{\gamma}}$. The functions $\Phi^{j}$ give rise to eigenfunctions of the Laplacian, provided that the factor $\phi_{\mu \bar{\mu}}^{j}$ is an eigenfunction of the operator

$$
\Delta_{\mu}=\frac{1}{4} \partial_{\phi}^{2}-\frac{1}{4} \partial_{\phi}-e^{2 \phi} \mu \bar{\mu}-\frac{1}{2} e^{\phi} \Xi \quad \text { where } \quad \Xi_{\mu}=\left(\partial_{\theta}-\theta \mu\right)\left(\partial_{\bar{\theta}}+\bar{\theta} \bar{\mu}\right) .
$$


Here and in the following we shall assume that $|\mu|^{2} \equiv \mu \bar{\mu}>0$. Our second step now is to diagonalize the operator $\Xi_{\mu}$ on the 4-dimensional Grassmann algebra that is generated from $\theta$ and $\bar{\theta}$. We can easily find two eigenfunctions $S_{\mu}^{ \pm}, T_{\mu}^{ \pm}$for each of the eigenvalues $\xi_{\mu}= \pm|\mu|$,

$$
\begin{aligned}
S_{\mu}^{+}(\theta, \bar{\theta}) & =\frac{1}{\sqrt{2}}(1-|\mu| \theta \bar{\theta}), & S_{\mu}^{-}(\theta, \bar{\theta}) & =\frac{1}{\sqrt{2}}(1+|\mu| \theta \bar{\theta}), \\
T_{\mu}^{+}(\theta, \bar{\theta}) & =\frac{1}{\sqrt{2}}(\sqrt{\mu} \theta-\sqrt{\bar{\mu}} \bar{\theta}), & T_{\mu}^{-}(\theta, \bar{\theta}) & =\frac{1}{\sqrt{2}}(\sqrt{\mu} \theta+\sqrt{\bar{\mu}} \bar{\theta}) .
\end{aligned}
$$

Having solved the eigenvalue problem for the operator $\Xi_{\mu}$, we can now split off the dependence of the eigenfunctions $\phi_{\mu \bar{\mu}}^{j}$ on fermionic coordinates through the following ansatz

$$
\phi_{\mu \bar{\mu}}^{j, \pm}(\theta, \bar{\theta}, \phi)=S_{\mu}^{ \pm}(\theta, \bar{\theta}) U_{p}^{ \pm}(\rho), \quad \psi_{\mu \bar{\mu}}^{j, \pm}(\theta, \bar{\theta}, \phi)=T_{\mu}^{ \pm}(\theta, \bar{\theta}) U_{p}^{ \pm}(\rho)
$$

Here we have introduced the letter $\psi$ instead of $\phi$ for odd eigenfunctions. Furthermore, the variable $\rho$ on the right hand side is defined by $\rho=2|\mu| e^{\phi}$. A short computation shows that the functions $U_{p}^{ \pm}$possess no explicit $\mu$ dependence any more. There remains a dependence on the parameter $j=-3 / 4+i p / 2$ which we display explicitly through the subscript $p$. In any case, the functions $\phi_{\mu \bar{\mu}}^{j, \pm}$ and $\psi_{\mu \bar{\mu}}^{j, \pm}$ give rise to eigenfunctions of our Laplacian provided that $U_{p}^{ \pm}(\rho)$ satisfy the second order differential equation

$$
\left(\partial_{\rho}^{2}-1 \mp \frac{1}{\rho}+\frac{1 / 4+p^{2}}{\rho^{2}}\right) U_{p}^{ \pm}(\rho)=0 .
$$

Solutions to these equations are

$$
U_{p}^{ \pm}(\rho)=\frac{\mathcal{C}^{i p}}{\Gamma\left(\frac{1}{2}-i p\right)}\left(\rho K_{\frac{1}{2}+i p}(\rho) \mp \rho K_{\frac{1}{2}-i p}(\rho)\right)
$$

In order to single out some unique solution we demanded regularity at $|\rho| \rightarrow \infty$. The normalization constant $\mathcal{C}$ remains undetermined for now.

In summary we have obtained a basis of eigenfunctions of the Laplacian $\Delta$ in $\operatorname{OSP}(1 \mid 2)$. Grassmann even functions with eigenvalue $\lambda=\lambda_{p}=(j+1)\left(j+\frac{1}{2}\right)=-\frac{1}{4}\left(\frac{1}{4}+p^{2}\right)$ take the form

$$
\Phi_{\mu \bar{\mu}}^{j, \pm}(\gamma, \bar{\gamma}, \theta, \bar{\theta}, \phi)=e^{\mu \gamma-\bar{\mu} \bar{\gamma}} S_{\mu}^{ \pm}(\theta, \bar{\theta}) U_{p}^{ \pm}\left(2|\mu| e^{\phi}\right)
$$

where $\mu, \bar{\mu}$ run through the complex plane. The functions $U_{p}^{ \pm}$and $S_{\mu}^{ \pm}$have been defined in eqs. (3.9) and (3.5), respectively. A similar formula with $T_{\mu}^{ \pm}$instead of $S_{\mu}^{ \pm}$holds for Grassmann odd eigenfunctions $\Psi_{\mu \bar{\mu}}^{j, \pm}$.

Let us conclude this subsection by computing the analogue of the minisuperspace 2-point function for Grassmann even functions in the $\operatorname{OSP}(1 \mid 2)$ model. ${ }^{3}$ This requires

\footnotetext{
${ }^{3} 2$-point functions for Grassmann odd functions $\Psi_{\mu, \bar{\mu}}^{j, \epsilon}$ can be computed in the same way. We will not discuss those here, neither in the particle model nor in the field theory.
} 
integrating the product of two functions (3.10) over the supergroup $\operatorname{OSP}(1 \mid 2)$. Using the Haar measure $[d g]=e^{-\phi} d \phi d \bar{\gamma} d \gamma d \bar{\theta} d \theta$ on $\operatorname{OSP}(1 \mid 2)$ we obtain

$$
\begin{aligned}
\left\langle\Phi_{\mu_{1}, \bar{\mu}_{1}}^{j_{1}, \epsilon_{1}} \Phi_{\mu_{2}, \bar{\mu}_{2}}^{j_{2}, \epsilon_{2}}\right\rangle_{0} & :=\int[d g] \Phi_{\mu_{1}, \bar{\mu}_{1}}^{j_{1}, \epsilon_{1}}(\gamma, \bar{\gamma}, \theta, \bar{\theta}, \phi) \phi_{\mu_{2} \bar{\mu}_{2}}^{j_{2}, \epsilon_{2}}(\gamma, \bar{\gamma}, \theta, \bar{\theta}, \phi) \\
& =-\left|\mu_{2}\right| \epsilon_{2} \delta_{\epsilon_{1}, \epsilon_{2}} \delta^{2}\left(\mu_{1}+\mu_{2}\right) \int \frac{d x}{x^{2}} U_{p_{1}}^{ \pm}\left(2\left|\mu_{1}\right| x\right) U_{p_{2}}^{ \pm}\left(2\left|\mu_{2}\right| x\right) \\
& =-2 \pi\left|\mu_{2}\right|^{2} \epsilon_{2} \delta_{\epsilon_{1}, \epsilon_{2}} \delta^{2}\left(\mu_{1}+\mu_{2}\right)\left(\delta\left(p_{1}+p_{2}\right)+\mathcal{R}^{\epsilon_{2}}\left(p_{2}\right) \delta\left(p_{1}-p_{2}\right)\right) .
\end{aligned}
$$

To get from the second to the third line we have utilized a formula for integrals of Bessel functions, see appendix B. The reflection amplitude $\mathcal{R}$ in the last line is given by

$$
\mathcal{R}^{ \pm}(p)=\mp \mathcal{C}^{2 i p} \frac{\Gamma\left(\frac{1}{2}+i p\right)}{\Gamma\left(\frac{1}{2}-i p\right)} .
$$

We shall later compare this answer with the outcome of a full fledged conformal field theory computation of the 2-point function. We could now start to analyze 3-point functions but before we do so, we would like to talk about another basis in the space of functions on $\operatorname{OSP}(1 \mid 2)$.

\subsection{Wave functions - another basis}

In the last subsection, we constructed a basis for the space of functions on $\operatorname{OSP}(1 \mid 2)$. This basis is very convenient for describing the duality between the $\operatorname{OSP}(1 \mid 2)$ WZNW model and $\mathcal{N}=1$ Liouville field theory. When it comes to writing down explicit formulas for correlation functions, on the other hand, there exists another, preferable choice. Recall that all correlators contain an osp $(1 \mid 2)$ invariant tensor that is determined by symmetries alone, along with the structure constants which contain all dynamical information. While the latter are the same in every basis, the former depend very much on our choices. We shall now present a new basis in which the osp(1|2) invariant tensors take a particularly simple form.

The transformation from the old basis (3.10) to the new can be thought of as a Fourier/Bessel transform in $\mu, \bar{\mu}$ and their fermionic counterparts. Let us perform the fermionic transformation first. This amounts to defining new functions $\Phi_{\mu}^{j}$ by

$$
\Phi_{\mu}^{j}(\xi \mid \gamma, \theta, \phi):=\frac{1}{|\mu|} \sum_{\epsilon= \pm}\left(S_{\mu}^{\epsilon}(\xi) \Phi_{\mu}^{j, \epsilon}(\gamma, \theta, \phi)+T_{\mu}^{\epsilon}(\xi) \Psi_{\mu}^{j, \epsilon}(\gamma, \theta, \phi)\right)
$$

Here and throughout the rest of this section we shall suppress spelling out the dependence on the bared quantities such as $\bar{\xi}, \bar{\theta}, \bar{\gamma}, \bar{\mu}$. Following [13], we also transform from the variables $\mu, \bar{\mu}$ to new variables $x, \bar{x}$,

$$
\Phi^{j}(x, \xi \mid \gamma, \theta, \phi):=\frac{1}{4 \pi}|\mu|^{-2 j-2} \int d^{2} \mu \Phi_{\mu}^{j}(\xi \mid \gamma, \theta, \phi) e^{\bar{\mu} \bar{x}-\mu x}
$$


We shall often refer to $\Phi^{j}(x, \xi \mid \gamma, \theta, \phi)$ as eigenfunctions in the $(x, \xi)$-basis. For given label $j$, the generators (3.1) of right translations may be expressed through their action on the auxiliary variables $x, \bar{x}, \xi, \bar{\xi}$ as follows,

$$
\begin{aligned}
& r_{E^{+}}=\partial_{x}, \quad r_{H}=-x \partial_{x}-\frac{1}{2} \xi \partial_{\xi}+j+\frac{1}{2}, \\
& r_{E^{-}}=-x^{2} \partial_{x}-x \xi \partial_{\xi}+x(2 j+1) \\
& r_{F^{+}}=\frac{1}{2}\left(\partial_{\xi}+\xi \partial_{x}\right), \quad r_{F^{-}}=-\frac{1}{2} x\left(\partial_{\xi}+\xi \partial_{x}\right)+\xi\left(j+\frac{1}{2}\right) .
\end{aligned}
$$

Given these expressions it is rather straightforward to verify that $\operatorname{osp}(1 \mid 2)$-invariance fixes the minisuperspace 2-point function to be of the form

$$
\left\langle\Phi^{j_{1}}\left(x_{1}, \xi_{1}\right) \Phi^{j_{2}}\left(x_{2}, \xi_{2}\right)\right\rangle=\frac{\pi}{4}\left[\delta^{2}\left(X_{12}\right) \delta\left(j_{1}+j_{2}+3 / 2\right)+\frac{B\left(j_{2}\right)}{\left|X_{12}\right|^{-4 j_{2}-2}} \delta\left(j_{1}-j_{2}\right)\right],
$$

up to an overall normalization which we have fixed such that the coefficient of $\delta$ functions in the first term is $\pi / 4$. In the first term we have also used the shorthand

$$
\delta^{2}\left(X_{12}\right)=\delta^{2}\left(x_{12}\right)\left(\xi_{1}-\xi_{2}\right)\left(\bar{\xi}_{1}-\bar{\xi}_{2}\right) .
$$

Furthermore, we employed the notation $X_{i j}=x_{i}-x_{j}-\xi_{i} \xi_{j}$ that will appear frequently throughout the rest of this note. The non-trivial structure constant $B(j)$ is not determined by symmetry. It may be calculated by explicitly performing the integral over the group manifold. Here, we shall follow a slightly different route. Our aim is to relate the two formulas (3.11) and (3.16) for the 2-point functions. We can then read off $B(j)$ from our formula (3.12) for the reflection amplitude $\mathcal{R}^{ \pm}(p)$. With the help of some integral formulas that are spelled out in appendix B, one may show that

$$
\left\langle\Phi_{\mu_{1}}^{j_{1}, \epsilon_{1}} \Phi_{\mu_{2}}^{j_{2}, \epsilon_{2}}\right\rangle_{0}=-\pi\left|\mu_{2}\right|^{2} \epsilon_{2} \delta_{\epsilon_{1}, \epsilon_{2}} \delta^{2}\left(\mu_{1}+\mu_{2}\right)\left[\delta\left(j_{1}+j_{2}+\frac{3}{2}\right)-\epsilon_{2} B\left(j_{2}\right) \pi \gamma\left(2 j_{2}+1\right) \delta\left(j_{1}-j_{2}\right)\right]
$$

where $\gamma(x)=\Gamma(x) / \Gamma(1-x)$. Indeed, this outcome is fully consistent with our previous formula (3.11). The comparison also allows us to determine the structure constant $B$,

$$
B(j)=-\frac{\epsilon \mathcal{R}^{\epsilon}(p)}{\pi \gamma(2 j+1)}=-\frac{1}{\pi} \mathcal{C}^{2 i p}
$$

where $j$ and $p$ are related by $j=-3 / 4+i p / 2$, as usual. By comparing the two expressions (3.16) and (3.11) for the 2-point function we have confirmed that the expression (3.11) is consistent with $\operatorname{osp}(1 \mid 2)$ invariance and we have determined the structure constant $B(j)$ that was introduced in eq. (3.16).

\subsection{The minisuperspace 3 -point function}

We are now prepared to move on to the analogue of the 3-point function in the particle model. Once more, the symmetry under osp $(1 \mid 2)$ transformation fixed the 3-point function up to two structure constants. In the $(x, \xi)$-basis, it reads

$$
\left\langle\Phi^{j_{1}}\left(x_{1}, \xi_{1}\right) \Phi^{j_{2}}\left(x_{2}, \xi_{2}\right) \Phi^{j_{3}}\left(x_{3}, \xi_{3}\right)\right\rangle_{0}=\frac{C\left(j_{1}, j_{2}, j_{3}\right)+\tilde{C}\left(j_{1}, j_{2}, j_{3}\right) \eta \bar{\eta}}{\left|X_{12}\right|^{-2 j_{12}-1}\left|X_{23}\right|^{-2 j_{23}-1}\left|X_{31}\right|^{-2 j_{31}-1}},
$$


where $j_{12}=j_{1}+j_{2}-j_{3}$ etc. and $X_{i j}=x_{i}-x_{j}-\xi_{i} \xi_{j}$, as before. Furthermore, the so-called super-projective 3 -point invariants $\eta, \bar{\eta}$ are

$$
\eta=\left(x_{12} x_{23} x_{31}\right)^{-\frac{1}{2}}\left(x_{23} \xi_{1}+x_{31} \xi_{2}+x_{12} \xi_{3}-\frac{1}{2} \xi_{1} \xi_{2} \xi_{3}\right)
$$

and similarly for $\bar{\eta} \cdot{ }^{4}$ The coefficients $C, \tilde{C}$ cannot be determined from osp(1|2) invariance. Instead, they require to perform the full integral over the supergroup manifold. Since we do not need the results, we are not going to compute the minisuperspace coefficients $C$ and $\tilde{C}$ explicitly. Determining their field theoretic analogue is one of the main issues in the next section.

Before we conclude this section, we would like to deduce from eq. (3.19) the 3-point function for the even part $\Phi^{\mathrm{B}}$ of our functions (3.13) in the mixed basis,

$$
\Phi_{\mu}^{\mathrm{B}, j}(\xi \mid \gamma, \theta, \phi):=\left(\Phi_{\mu}^{j}(\xi \mid \gamma, \theta, \phi)\right)^{\text {even }}=\frac{1}{|\mu|} \sum_{\epsilon= \pm} S_{\mu}^{\epsilon}(\xi) \Phi_{\mu}^{j, \epsilon}(\gamma, \theta, \phi)
$$

The field theoretic analogues of these functions shall feature in the next section when we discuss correlators of the OSP(1|2) WZNW model. A short and straightforward computation shows that

$$
\begin{aligned}
\left\langle\prod_{i=1}^{3} \Phi_{\mu_{i}, \bar{\mu}_{i}}^{\mathrm{B}, j_{i}}\left(\xi_{i}\right)\right\rangle_{0} & =C\left(j_{1}, j_{2}, j_{3}\right)\left(D_{0}\left[j_{i}, \mu_{i}\right]+\frac{1}{2} \sum_{a, b, c=1}^{3} \epsilon_{a b c} D_{b c}\left[j_{i}, \mu_{i}\right] \xi_{b} \bar{\xi}_{b} \xi_{c} \bar{\xi}_{c}\right) \\
& +\tilde{C}\left(j_{1}, j_{2}, j_{3}\right)\left(\sum_{a=1}^{3} D_{a}\left[j_{i}, \mu_{i}\right] \xi_{a} \bar{\xi}_{a}+D_{123}\left[j_{i}, \mu_{i}\right] \xi_{1} \bar{\xi}_{1} \xi_{2} \bar{\xi}_{2} \xi_{3} \bar{\xi}_{3}\right)
\end{aligned}
$$

where $C$ and $\tilde{C}$ are the same as before and $\epsilon_{123}=1$ and so on. Various group theoretic factors are given by

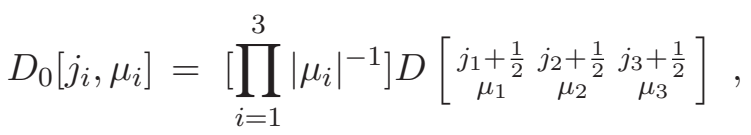

$$
\begin{aligned}
& D_{123}\left[j_{i}, \mu_{i}\right]=-(j+2)^{2} D\left[\begin{array}{lll}
j_{1} & j_{2} & j_{3} \\
\mu_{1} & \mu_{2} & \mu_{3}
\end{array}\right], \\
& D_{1}\left[j_{i}, \mu_{i}\right]=\left|\mu_{2}\right|^{-1}\left|\mu_{3}\right|^{-1} D\left[\begin{array}{ccc}
j_{1} & j_{2}+\frac{1}{2} & j_{3}+\frac{1}{2} \\
\mu_{1} & \mu_{2} & \mu_{3}
\end{array}\right], \\
& D_{12}\left[j_{i} \mu_{i}\right]=-\left|\mu_{3}\right|^{-1}\left(j_{12}+\frac{1}{2}\right)^{2} D\left[\begin{array}{ccc}
j_{1} & j_{2} & j_{3}+\frac{1}{2} \\
\mu_{1} & \mu_{2} & \mu_{3}
\end{array}\right],
\end{aligned}
$$

and those with index permutations. On the right hand side there appears the single new function $D$ that is defined in (B.7). As we anticipated, the final expression for the 3-point function in the $\mu$-basis turns out to be rather involved simply because the group theoretic contributions to the structure constants are rather complicated.

\footnotetext{
${ }^{4}$ Since the osp $(1 \mid 2)$ superalgebra has super dimension $3 \mid 2$, we can fix three bosonic parameters $x$ and two fermionic parameters $\xi$. Therefore, the three point function still depends on a fermionic invariant $\eta$.
} 


\section{Solution of the $\operatorname{OSP}(1 \mid 2)$ WZNW model}

In this section we compute the structure constants of OSP(1|2) WZNW model. As we discussed in the previous section, the 2- and 3-point functions are almost fixed by $\operatorname{osp}(1 \mid 2)$ symmetry, up to three coefficients $B_{b}, C_{b}, \tilde{C}_{b}$ that remain undetermined and turn out to acquire field theoretic modifications. In order to fix these coefficients we utilize the results of section 2, where we have derived the relation between correlators of $\operatorname{OSP}(1 \mid 2)$ model and $\mathcal{N}=1$ super Liouville theory.

\subsection{The WZNW-Liouville correspondence revisited}

When we discussed the correspondence between the OSP(1|2) WZNW model and $\mathcal{N}=1$ Liouville theory we worked with vertex operators $V_{j}^{\epsilon, \bar{\epsilon}}(\mu \mid z)$ containing both bosonic and fermionic components. In our computations here we shall restrict ourselves to correlators involving purely bosonic fields since they are enough to fix the unknown functions. The bosonic component of $V_{j}^{\epsilon, \bar{\epsilon}}(\mu \mid z)$ is a field theoretic analogue of the function (3.10) on $\operatorname{OSP}(1 \mid 2)$, i.e.

$$
V_{j}^{\epsilon}(\mu \mid z):=\frac{1}{\sqrt{2}}\left(V_{j}^{\epsilon^{\prime}, \bar{\epsilon}^{\prime}}(\mu \mid z)\right)^{\text {even }}=\frac{1}{\sqrt{2}}(1-\epsilon|\mu| \theta \bar{\theta})|\mu|^{2 j+2} e^{\mu \gamma-\bar{\mu} \bar{\gamma}} e^{2 b(j+1) \phi}
$$

where $\epsilon=-\epsilon^{\prime} \bar{\epsilon}^{\prime}$. Note that $V_{j}^{\epsilon}(\mu \mid z)$ are indeed modeled after the functions (3.10), i.e.

$$
V_{j}^{ \pm}(\mu \mid z)=S_{\mu}^{ \pm}(\theta, \bar{\theta}) e^{\mu \gamma-\bar{\mu} \bar{\gamma}}|\mu|^{2 j+1} e^{2(j+1) \phi}
$$

where $S_{\mu}^{ \pm}$is the same as in eq. (3.5). Only now the symbol $\theta=\theta(z)$ denotes a fermionic field on the world-sheet and similarly for $\bar{\theta}$. Under the change of variables described in section 2 , the field $S_{\mu}^{ \pm}$becomes

$$
S_{\mu}^{ \pm}=\frac{1}{\sqrt{2}}\left(1 \pm i \theta^{\prime} \bar{\theta}^{\prime}\right)=\frac{1}{\sqrt{2}}\left(1 \pm \frac{i}{2}(\psi-i \chi)(\bar{\psi}+i \bar{\chi})\right)=\frac{1}{\sqrt{2}}\left(1 \pm e^{-i Y+i \bar{Y}}\right) .
$$

With this preparation we can now compute correlation functions of the fields $V_{j}^{\epsilon}(\mu \mid z)$ in the $\operatorname{OSP}(1 \mid 2)$ WZNW model through our relation (2.21) with $\mathcal{N}=1$ Liouville field theory,

$$
\left\langle\prod_{\nu=1}^{N} V_{j_{\nu}}^{\epsilon_{\nu}}\left(\mu_{\nu} \mid z_{\nu}\right)\right\rangle=\delta^{2}\left(\sum_{\nu=1}^{N} \mu_{\nu}\right)\left|\Theta_{N}^{(1)}\right|^{2}\left\langle\prod_{\nu=1}^{N} S^{\epsilon_{\nu}} e^{\alpha_{\nu} \varphi}\left(z_{\nu}\right) \prod_{j=1}^{N-2} e^{-\frac{i}{2} \mathcal{Y}} e^{-\frac{1}{2 b} \varphi}\left(y_{j}\right)\right\rangle^{L}
$$

with $b=1 / \sqrt{2 k-3}$ and $\alpha_{\nu}=2 b\left(j_{\nu}+1\right)+1 / 2 b$. The index ${ }^{L}$ on the right hand side reminds us that the correlator is to be computed in the product of super Liouville theory with a free fermion theory. Here we have defined

$$
S^{+}=\sqrt{2} \cos \frac{\mathcal{Y}}{2}, \quad S^{-}=\sqrt{2} i \sin \frac{\mathcal{Y}}{2}, \quad \mathcal{Y}=Y-\bar{Y} .
$$

Since the fields $S^{ \pm}$include both the fermionic field $\chi$ of the free fermion theory and the fermion $\psi$ of $\mathcal{N}=1$ Liouville theory, it is not straightforward to apply the results of $\mathcal{N}=1$ 
super Liouville field theory. In order to do so we utilize the well-known construction of $S^{ \pm}$ through spin fields of the real fermions (see e.g. [26]),

$$
\begin{aligned}
& \left\langle\prod_{i=1}^{2 m} S^{+}\left(z_{i}\right) \prod_{j=2 m+1}^{2 n} S^{-}\left(z_{j}\right)\right\rangle= \\
& \quad=(-1)^{n-m}\left\langle\prod_{i=1}^{2 m} \Sigma_{\chi}^{+}\left(z_{i}\right) \prod_{j=2 m+1}^{2 n} \Sigma_{\chi}^{-}\left(z_{j}\right)\right\rangle\left\langle\prod_{i=1}^{2 m} \Sigma_{\psi}^{+}\left(z_{i}\right) \prod_{j=2 m+1}^{2 n} \Sigma_{\psi}^{-}\left(z_{j}\right)\right\rangle,
\end{aligned}
$$

where $\Sigma_{\chi}^{ \pm}$and $\Sigma_{\psi}^{ \pm}$are spin fields for the real fermions $\chi$ and $\psi$, respectively.

\subsection{Computation of 2-point functions}

In order to practice using our relation (4.2), we want to compute the 2-point function of OSP(1|2) WZNW model. This case is rather simple since no extra degenerate fields are to be inserted. With eq. (4.2) and eq. (4.4) we have

$$
\begin{aligned}
& \left\langle V_{j_{1}}^{\epsilon_{1}}\left(\mu_{1} \mid z_{1}\right) V_{j_{2}}^{\epsilon_{2}}\left(\mu_{2} \mid z_{2}\right)\right\rangle= \\
& \quad=\delta^{2}\left(\mu_{1}+\mu_{2}\right)|u|^{2}\left|z_{12}\right|^{\frac{1}{2 b^{2}}-\frac{1}{2}}\left\langle S^{\epsilon_{1}} e^{\alpha_{1} \varphi}\left(z_{1}\right) S^{\epsilon_{2}} e^{\alpha_{2} \varphi}\left(z_{2}\right)\right\rangle^{L} \\
& \quad=-i e^{\frac{\pi i}{4}\left(\epsilon_{1}+\epsilon_{2}\right)} \delta^{2}\left(\mu_{1}+\mu_{2}\right)|u|^{2}\left|z_{12}\right|^{\frac{1}{2 b^{2}}-\frac{1}{2}}\left\langle\Sigma_{\chi}^{\epsilon_{1}}\left(z_{1}\right) \Sigma_{\chi}^{\epsilon_{2}}\left(z_{2}\right)\right\rangle\left\langle\Sigma_{\alpha_{1}}^{\epsilon_{1}}\left(z_{1}\right) \Sigma_{\alpha_{2}}^{\epsilon_{2}}\left(z_{2}\right)\right\rangle
\end{aligned}
$$

where $\Sigma_{\alpha}^{ \pm}$are spin fields in $\mathcal{N}=1$ Liouville theory, see eq. (C.9) for a definition. Inserting the two point function of spin fields in the free fermion theory,

$$
\left\langle\Sigma_{\chi}^{\epsilon_{1}}\left(z_{1}\right) \Sigma_{\chi}^{\epsilon_{2}}\left(z_{2}\right)\right\rangle=\delta_{\epsilon_{1}, \epsilon_{2}}\left|z_{12}\right|^{-\frac{1}{4}},
$$

along with the corresponding formula for the 2-point function of $\Sigma_{\alpha}^{ \pm}$in super Liouville theory, see eq. (C.10), the 2-point function of OSP(1|2) WZNW model can be evaluated as

$$
\left\langle V_{j_{1}}^{\epsilon_{1}}\left(\mu_{1} \mid z_{1}\right) V_{j_{2}}^{\epsilon_{2}}\left(\mu_{2} \mid z_{2}\right)\right\rangle=\delta_{\epsilon_{2}}^{\epsilon_{1}} \delta^{2}\left(\mu_{1}+\mu_{2}\right) \frac{\pi\left|\mu_{2}\right|^{2} \epsilon_{2}}{b\left|z_{12}\right|^{4 \Delta_{j_{2}}}}\left[\delta\left(j_{1}+j_{2}+\frac{3}{2}\right)-\epsilon_{2} \delta\left(j_{1}-j_{2}\right) D_{R}^{L}\left(\alpha_{2}\right)\right]
$$

with $\Delta_{j}=-2 b^{2}(j+1)\left(j+\frac{1}{2}\right)$. An explicit formula for the structure functions $D_{R}^{L}$ of Liouville theory may be found in eq. (C.11). By comparing our result with the general form of the 2-point invariant (3.16) we read off that

$$
B_{b}(j)=\frac{D_{R}^{L}\left(2 b(j+1)+\frac{1}{2 b}\right)}{\pi \gamma(2 j+1)}=-\frac{1}{\pi}\left(\frac{2 k b^{2}}{i \gamma\left(\frac{b^{2}+1}{2}\right)}\right)^{4 j+3} \frac{\Gamma\left(\frac{1}{2}+b^{2}\left(2 j+\frac{3}{2}\right)\right)}{\Gamma\left(\frac{1}{2}-b^{2}\left(2 j+\frac{3}{2}\right)\right)} .
$$

In the limit $b \rightarrow 0$ we recover the result (3.18) of the particle model.

\subsection{Computation of 3-point functions}

Our aim now is to determine the structure constants of the 3-point function in the $\operatorname{OSP}(1 \mid 2)$ WZNW model from the correspondence with $\mathcal{N}=1$ Liouville theory. To this end we 
compute the 3-point function of three bosonic vertex operators (4.1) using the formula (4.2).

$$
\begin{aligned}
\left\langle V_{j_{1}}^{\epsilon_{1}}\left(\mu_{1} \mid z_{1}\right) V_{j_{2}}^{\epsilon_{2}}\right. & \left.\left(\mu_{2} \mid z_{2}\right) V_{j_{3}}^{\epsilon_{3}}\left(\mu_{3} \mid z_{3}\right)\right\rangle=\delta^{2}\left(\mu_{1}+\mu_{2}+\mu_{3}\right)\left|\Theta_{3}^{(1)}\right|^{2} \times \\
& \times\left\langle\frac{1}{\sqrt{2}}\left(S^{+}+S^{-}\right) e^{-\frac{1}{2 b} \varphi}(y) S^{\epsilon_{1}} e^{\alpha_{1} \varphi}\left(z_{1}\right) S^{\epsilon_{2}} e^{\alpha_{2} \varphi}\left(z_{2}\right) S^{\epsilon_{3}} e^{\alpha_{3} \varphi}\left(z_{3}\right)\right\rangle^{L} .
\end{aligned}
$$

Here, we use the same notations as in eq. (4.2) before. Note that the computation of a 3-point function on the OSP(1|2) WZNW model requires one additional insertion of a degenerate Liouville field in the correlator on the right hand side. This field is inserted at

$$
y=-\frac{1}{u}\left(\mu_{1} z_{2} z_{3}+\mu_{2} z_{3} z_{1}+\mu_{3} z_{1} z_{2}\right)
$$

where the parameter $u$ is given by $u=\sum_{i=1}^{3} \mu_{i} z_{i}$. Furthermore, for $N=3$ the twist factor $\left|\Theta_{3}^{(1)}\right|^{2}$ defined in eq. (2.22) simplifies as follows

$$
\left|\Theta_{3}^{(1)}\right|^{2}=|u|^{\frac{3}{2 b^{2}}+\frac{1}{2}} \prod_{i<j}\left|z_{i j}\right|^{-\frac{1}{2 b^{2}}+\frac{1}{2}} \prod_{i=1}^{3}\left|\mu_{i}\right|^{-\frac{1}{2 b^{2}}+\frac{1}{2}} .
$$

As in the case of the 2-point function we can express the fields $S^{ \pm}$in terms of twist fields for the two fermions using the formula (4.4). The correlator of four twist fields in a free fermion model is known from the work of Belavin, Polyakov and Zamolodchikov [27],

$$
\left\langle\Sigma_{\chi}^{\epsilon_{0}}\left(z_{0}\right) \Sigma_{\chi}^{\epsilon_{1}}\left(z_{1}\right) \Sigma_{\chi}^{\epsilon_{2}}\left(z_{2}\right) \Sigma_{\chi}^{\epsilon_{3}}\left(z_{3}\right)\right\rangle=\left|z_{03}\right|^{-\frac{1}{4}}\left|z_{12}\right|^{-\frac{1}{4}} \mathcal{I}^{\epsilon_{0} \epsilon_{1} \epsilon_{2} \epsilon_{3}}(z) .
$$

Here $z\left(\equiv\left(z_{01} z_{23}\right) /\left(z_{03} z_{21}\right)\right)=1+\mu_{2} / \mu_{3}$ is the conformally invariant cross ratio of the points $z_{0}=y$ and $z_{i}, i=1,2,3$. The functions $\mathcal{I}^{\epsilon_{0} \epsilon_{1} \epsilon_{2} \epsilon_{3}}(z)$ are given by

$$
\begin{array}{ll}
\mathcal{I}^{ \pm \pm \pm \pm}(z)=I_{0}(z) I_{0}(\bar{z})+I_{\frac{1}{2}}(z) I_{\frac{1}{2}}(\bar{z}), & \mathcal{I}^{ \pm \pm \mp \mp}(z)=I_{0}(z) I_{0}(\bar{z})-I_{\frac{1}{2}}(z) I_{\frac{1}{2}}(\bar{z}), \\
\mathcal{I}^{ \pm \mp \pm \mp}(z)= \pm\left[I_{0}(z) I_{\frac{1}{2}}(\bar{z})+I_{\frac{1}{2}}(z) I_{0}(\bar{z})\right], \mathcal{I}^{ \pm \mp \mp \pm}(z)=i\left[I_{0}(z) I_{\frac{1}{2}}(\bar{z})-I_{\frac{1}{2}}(z) I_{0}(\bar{z})\right],
\end{array}
$$

with

$$
I_{0}(z)=(z(1-z))^{-\frac{1}{8}} F\left(\frac{1}{4},-\frac{1}{4}, \frac{1}{2}, z\right), \quad I_{\frac{1}{2}}(z)=\frac{1}{2}(z(1-z))^{\frac{3}{8}} F\left(\frac{5}{4}, \frac{3}{4}, \frac{3}{2}, z\right) .
$$

As for the contribution from Liouville theory, all relevant formulas are listed in appendix C. The relevant 4-point function (C.20) was constructed in [20]. It involves a new function $\mathcal{H}^{\epsilon_{0} \epsilon_{1} \epsilon_{2} \epsilon_{3}}$ that we spell out explicitly in eq. (C.24). Putting all these pieces together we obtain

$$
\begin{aligned}
& \left\langle V_{j_{1}}^{\epsilon_{1}}\left(\mu_{1} \mid z_{1}\right) V_{j_{2}}^{\epsilon_{2}}\left(\mu_{2} \mid z_{2}\right) V_{j_{3}}^{\epsilon_{3}}\left(\mu_{3} \mid z_{3}\right)\right\rangle=\delta^{2}\left(\mu_{1}+\mu_{2}+\mu_{3}\right) \prod_{i<j}\left|z_{i j}\right|^{-2 \Delta_{i j}} \times \\
& \quad \times\left|\mu_{1}\right|^{-\frac{1}{2 b^{2}}+\frac{1}{2}}\left|\mu_{2}\right|^{-\frac{1}{2 b^{2}}+\frac{1}{2}}\left|\mu_{3}\right|^{\frac{1}{b^{2}}+1} \frac{e^{\pi i\left(4-\epsilon-\sum \epsilon_{\nu}\right) / 4}}{\sqrt{2}} \mathcal{I}^{\epsilon \epsilon_{1} \epsilon_{2} \epsilon_{3}}\left(1+\frac{\mu_{2}}{\mu_{3}}\right) \mathcal{H}^{\epsilon \epsilon_{1} \epsilon_{2} \epsilon_{3}}\left(1+\frac{\mu_{2}}{\mu_{3}}\right),
\end{aligned}
$$

where $\epsilon=\epsilon_{1} \epsilon_{2} \epsilon_{3}$. In principle we have thereby completed our computation of the 3-point function in the OSP(1|2) WZNW model. Of course, in its present form the answer is not 
very illuminating, in particular when compared with the relatively simple form of the 3point function we anticipated in eq. (1.1) of the introduction. The reason our formula (4.13) looks somewhat unfamiliar was discussed in detail in section 3.3: It is the transformation from the $x$ to the $\mu$ basis that turns the rather simple looking formulas (3.19) or (1.1) into the bulky expression of eqs. (3.22) or (4.13). Our final task is therefore to perform the transformation from eq. (4.13) to (1.1). We shall not discuss this in full detail but simply look at two of the terms in eq. (3.22) which suffice to read off the structure functions $C_{b}$ and $\tilde{C}_{b}$.

Let us begin with the coefficient $\tilde{C}_{b}$ in eq. (1.1). Comparison with our minisuperspace formula (3.22) shows that $\tilde{C}$ appears in the coefficient of the term with the maximal number of Grassmann variables. In fact, the coefficient of this term is a product of $\tilde{C}$ with the group theoretic factor $D_{123}$. In order to compare with our field theoretic outcome, we switch from the $(\mu, \epsilon)$ basis to the mixed basis involving $\mu$ and $\xi$, i.e. we rewrite the correlation function (4.13) in terms of the fields

$$
V_{j}^{\mathrm{B}}(\mu, \xi \mid z):=\left(V_{j}(\mu, \xi \mid z)\right)^{\mathrm{even}}=\frac{1}{|\mu|} \sum_{\epsilon= \pm} S_{\mu}^{\epsilon}(\xi) V_{j}^{\epsilon}(\mu \mid z)
$$

The definition of $V^{\mathrm{B}}$ is modeled after the construction (3.21) in the particle theory. From the discussion above we infer that

$$
\begin{aligned}
& \tilde{C}_{b}\left(j_{1}, j_{2}, j_{3}\right) D_{123}\left[j_{i}, \mu_{i}\right]= \\
& =\lim _{z_{\infty} \rightarrow \infty} \int \prod_{i=1}^{3} d \bar{\xi}_{i} d \xi_{i}\left|z_{\infty}\right|^{4 \Delta_{j_{1}}}\left\langle V_{j_{1}}^{\mathrm{B}}\left(\mu_{1}, \xi_{1} \mid z_{\infty}\right) V_{j_{2}}^{\mathrm{B}}\left(\mu_{2}, \xi_{2} \mid 1\right) V_{j_{3}}^{\mathrm{B}}\left(\mu_{3}, \xi_{3} \mid 0\right)\right\rangle \\
& =\lim _{z_{\infty} \rightarrow \infty} \frac{1}{2 \sqrt{2}} \sum_{\epsilon_{i}= \pm}\left(-\epsilon_{1} \epsilon_{2} \epsilon_{3}\right)\left|z_{\infty}\right|^{4 \Delta_{j_{1}}}\left\langle V_{j_{1}}^{\epsilon_{1}}\left(\mu_{1} \mid z_{\infty}\right) V_{j_{2}}^{\epsilon_{2}}\left(\mu_{2} \mid 1\right) V_{j_{3}}^{\epsilon_{3}}\left(\mu_{3} \mid 0\right)\right\rangle .
\end{aligned}
$$

Now we need to insert our result (4.13) along with formulas for $\mathcal{I}$ and $\mathcal{H}$. After that we can compute the sum on the right hand side of the previous equation. It is easy to see that all terms involving the Liouville structure constant $\tilde{C}_{R}^{L}$ cancel from the resulting expression. The terms proportional to $C_{R}^{L}$ are determined with the help of the following auxiliary formula

$$
\begin{aligned}
& \left(G\left(b p_{1}, b p_{2}, b p_{3} ; z\right)+\sqrt{1-z} G\left(b p_{1},-b p_{2},-b p_{3} ; z\right)\right) \times \\
& \quad \times\left(G\left(b p_{1}, b p_{2}, b p_{3} ; \bar{z}\right)+\sqrt{1-\bar{z}} G\left(b p_{1},-b p_{2},-b p_{3} ; \bar{z}\right)\right)= \\
& \quad=|z|^{\frac{1}{2 b^{2}}+i p_{1}}|1-z|_{2}^{\frac{1}{2 b^{2}}+i p_{2}} \mathcal{F}_{1}\left(-\frac{1}{4}+\frac{i}{2} p, \frac{1}{4}+\frac{i}{2} p_{12}, \frac{1}{2}+i p_{1} ; z\right)
\end{aligned}
$$

where $p_{12}=p_{1}+p_{2}-p_{3}$ etc. and $p=p_{1}+p_{2}+p_{3}$. The parameter $z$ takes the same value $z=1+\mu_{2} / \mu_{3}$ as before. In the derivation of the formula we have used the well known identities

$$
\frac{1}{2} \sin \kappa \cos \kappa F\left(\frac{5}{4}, \frac{3}{4}, \frac{3}{2}, \sin ^{2} \kappa\right)=\sin \frac{1}{2} \kappa, \quad F\left(\frac{1}{4},-\frac{1}{4}, \frac{1}{2}, \sin ^{2} \kappa\right)=\cos \frac{1}{2} \kappa,
$$


and

$$
(c-b-1) F(a, b, c ; z)+b(1-z) F(a, b+1, c ; z)=(c-1) F(a-1, b, c-1 ; z) .
$$

The resulting expression for the sum in eq. (4.15) is of the form $\tilde{C}_{b} D_{123}$ with $D_{123}$ given by formula (3.23) if the structure function $\tilde{C}\left(j_{1}, j_{2}, j_{3}\right)$ is introduced as

$$
\begin{aligned}
\tilde{C}_{b}\left(j_{1}, j_{2}, j_{3}\right) & =\frac{1}{2 \pi} C_{R}^{L}\left(\alpha_{1}-\frac{1}{2 b}, \alpha_{2}, \alpha_{3}\right) \frac{\gamma\left(\frac{1}{4}-\frac{i}{2} p\right) \gamma\left(\frac{1}{2}+i p_{1}\right)}{\gamma\left(\frac{1}{4}+\frac{i}{2} p_{31}\right) \gamma\left(\frac{1}{4}+\frac{i}{2} p_{12}\right)} \\
& =\frac{1}{2 \pi b}\left(\frac{2 k b^{2+b^{2}}}{i \gamma\left(\frac{b^{2}+1}{2}\right)}\right)^{2 j+5} \frac{\Upsilon_{\mathrm{NS}}^{\prime}(0)}{\Upsilon_{\mathrm{NS}}\left(2 b\left(j+\frac{5}{2}\right)+\frac{1}{b}\right)} \times \\
& \times \frac{\Upsilon_{\mathrm{R}}\left(4 b\left(j_{1}+1\right)+\frac{1}{b}\right) \Upsilon_{\mathrm{R}}\left(4 b\left(j_{2}+1\right)+\frac{1}{b}\right) \Upsilon_{\mathrm{R}}\left(4 b\left(j_{3}+1\right)+\frac{1}{b}\right)}{\Upsilon_{\mathrm{R}}\left(2 b\left(j_{12}+1\right)+\frac{1}{b}\right) \Upsilon_{\mathrm{R}}\left(2 b\left(j_{23}+1\right)+\frac{1}{b}\right) \Upsilon_{\mathrm{R}}\left(2 b\left(j_{31}+1\right)+\frac{1}{b}\right)} .
\end{aligned}
$$

A very similar analysis furnishes an expression for the structure constant $C_{b}\left(j_{1}, j_{2}, j_{3}\right)$. Another glance onto eq. (3.22) shows that $C_{b}$ may be determined e.g. from the terms proportional to $\xi_{1} \bar{\xi}_{1} \xi_{2} \bar{\xi}_{2}$ in the correlators of $V_{j}^{\mathrm{B}}(\mu, \xi \mid z)$,

$$
\begin{aligned}
& C_{b}\left(j_{1}, j_{2}, j_{3}\right) D_{12}\left[j_{i}, \mu_{i}\right] \\
& =\lim _{z_{\infty} \rightarrow \infty} \int \prod_{i=1}^{3} d \bar{\xi}_{i} d \xi_{i} \xi_{3} \bar{\xi}_{3}\left|z_{\infty}\right|^{4 \Delta_{j_{1}}}\left\langle V_{j_{1}}^{\mathrm{B}}\left(\mu_{1}, \xi_{1} \mid z_{\infty}\right) V_{j_{2}}^{\mathrm{B}}\left(\mu_{2}, \xi_{2} \mid 1\right) V_{j_{3}}^{\mathrm{B}}\left(\mu_{3}, \xi_{3} \mid 0\right)\right\rangle \\
& =\lim _{z_{\infty} \rightarrow \infty} \frac{1}{2 \sqrt{2}} \sum_{\epsilon_{i}= \pm} \frac{\epsilon_{1} \epsilon_{2}}{\left|\mu_{3}\right|}\left|z_{\infty}\right|^{4 \Delta_{j_{1}}}\left\langle V_{j_{1}}^{\epsilon_{1}}\left(\mu_{1} \mid z_{\infty}\right) V_{j_{2}}^{\epsilon_{2}}\left(\mu_{2} \mid 1\right) V_{j_{3}}^{\epsilon_{3}}\left(\mu_{3} \mid 0\right)\right\rangle .
\end{aligned}
$$

The sum on the right hand side can be computed in the precisely the same way as before, and the result is given by replacing $p_{3}$ with $-p_{3}$ and $C_{R}^{L}$ with $\tilde{C}_{R}^{L}$. Thus we conclude

$$
\begin{aligned}
C_{b}\left(j_{1}, j_{2}, j_{3}\right) & =\frac{1}{2 \pi} \tilde{C}_{R}^{L}\left(\alpha_{1}-\frac{1}{2 b}, \alpha_{2}, \alpha_{3}\right) \frac{\gamma\left(\frac{3}{4}-\frac{i}{2} p\right) \gamma\left(\frac{1}{2}+i p_{1}\right)}{\gamma\left(\frac{3}{4}+\frac{i}{2} p_{31}\right) \gamma\left(\frac{3}{4}+\frac{i}{2} p_{12}\right)} \\
& =\frac{1}{2 \pi}\left(\frac{2 k b^{2+b^{2}}}{i \gamma\left(\frac{b^{2}+1}{2}\right)}\right)^{2 j+5} \frac{\Upsilon_{\mathrm{NS}}^{\prime}(0)}{\Upsilon_{\mathrm{R}}\left(2 b\left(j+\frac{5}{2}\right)+\frac{1}{b}\right)} \times \\
& \times \frac{\Upsilon_{\mathrm{R}}\left(4 b\left(j_{1}+1\right)+\frac{1}{b}\right) \Upsilon_{\mathrm{R}}\left(4 b\left(j_{2}+1\right)+\frac{1}{b}\right) \Upsilon_{\mathrm{R}}\left(4 b\left(j_{3}+1\right)+\frac{1}{b}\right)}{\Upsilon_{\mathrm{NS}}\left(2 b\left(j_{12}+1\right)+\frac{1}{b}\right) \Upsilon_{\mathrm{NS}}\left(2 b\left(j_{23}+1\right)+\frac{1}{b}\right) \Upsilon_{\mathrm{NS}}\left(2 b\left(j_{31}+1\right)+\frac{1}{b}\right)} .
\end{aligned}
$$

\section{Conclusion}

In this note we have solved the very simplest example of a WZNW model on a type II supergroup, namely on the supergroup $\operatorname{OSP}(1 \mid 2)$. Our discussion here was restricted to the NSNS sector of the theory but the analysis can easily be extended to the RR sector. The associated structure constants then involve the 2- and 3-point couplings in the NSNS sector of $\mathcal{N}=1$ Liouville theory. A more interesting problem would be to include boundary conditions into the analysis. According to [28] (see also [29] for a generalization to 
supergroups), maximally symmetric branes in the OSP(1|2) WZNW model correspond to (twisted) super-conjugacy classes. Under the OSP(1|2) WZNW super-Liouville correspondence, branes in the $\operatorname{OSP}(1 \mid 2)$ model should map to branes in $\mathcal{N}=1$ Liouville theory. The latter have been studied by several authors, see in particular [20,30]. In addition, it should also be possible to find a precise relation between correlation functions on the half-plane. In the case of the ordinary $H_{3}^{+}$-Liouville correspondence, such relations were found in [31] and rederived by means of the path integral approach in [32].

To the best of our knowledge, the OSP(1|2) WZNW model had not been solved previously, though it is certainly possible to find its 2- and 3-point couplings more directly, i.e. without the relation to supersymmetric Liouville theory, through the evaluation of factorization constraints. Such an approach has been successfully applied to the $\mathrm{H}_{3}^{+}$model in [10]. It would be interesting to generalize the analysis of factorization constraints to the OSP(1|2) WZNW model.

The proposal of Ribault and Teschner for the concrete relation between local correlators in $H_{3}^{+}$model and Liouville field theory emerged partly from a careful comparison of differential equations on both sides of the correspondence. The correlators of any WZNW model obey the famous Knizhnik-Zamolodchikov equations. On the Liouville side, one higher order differential equation arises from each degenerate field insertion. These two types of differential equations are mapped onto each other by the $\mathrm{H}_{3}^{+}$-Liouville correspondence, see $[13,16]$. A similar analysis for the relation between the OSP(1|2) WZNW model and $\mathcal{N}=1$ Liouville theory has not been performed yet.

There are various other extensions of our path integral approach that merit further study. Our basic strategy above was to apply the reduction ideas of [16] to the $\mathrm{sl}_{2}$ current algebra that resides within the osp $(1 \mid 2)$ current algebra of the WZNW model. As we have explained in the introduction and illustrated in section 2.2, the same concepts apply to more general type II supergroups. It might be interesting to work this out in more detail, in particular for supergroups $\operatorname{OSP}(p \mid \mathrm{N})$ with parameters $\mathrm{N} \geq 3$. Another obvious extension would be to solve the $\operatorname{OSP}(2 \mid 2)$ WZNW model through its relation with $\mathcal{N}=2$ Liouville field theory. While bulk 2-point functions of the latter model have been studied [33] and a conjecture for bulk 3-point functions was formulated in [34], higher correlators are not yet available. In this context, it may also be worthwhile investigating the precise relation between the OSP $(2 \mid 2)$ WZNW model discussed above and the SL(1|2) theory that has been solved in $[35,18]$. The $\operatorname{OSP}(2 \mid 2)$ WZNW model was also investigated in the condensed matter literature, see e.g. [36, 37] and references therein.

In the case of WZNW models on type I supergroups it is possible to solve them in terms of a purely bosonic model [18]. It seems likely that for type II supergroups a further reduction is possible in which the remaining fermionic fields are also removed. Indeed, for $\mathcal{N}=1$ Liouville field theory the structure constants are very closely related to those of the purely bosonic Liouville model. It would be rewarding to find a formal path integral derivation of this relation and to generalize it to higher supergroups.

Let us finally mention a rather different direction to which some of the above might apply. We have discussed in the introduction that correspondences of the proposed type 
elevate a usual Hamiltonian reduction to an equivalence between local field theories of different target space dimension. But Hamiltonian reduction also links WZNW models for groups of higher rank to certain conformal Toda theories, see e.g. [38]. It is indeed likely that $N$-point functions of tachyon vertex operators in WZNW models can be more generally related to correlators in Toda theory. Unfortunately, no explicit formulas have been derived yet. The main technical obstacle arises from the non-abelian nature of the maximal nilpotent subalgebra. In this sense, even the osp(1|2) case we have studied here could turn out to be a rather instructive example. The maximal nil-potent subalgebra of $\operatorname{osp}(1 \mid 2)$, i.e. the algebra spanned by $F^{+}$and $E^{+}$, is non-abelian. Hence an equivalence between the OSP(1|2) WZNW model and bosonic Liouville field theory (see previous paragraph) could be the first instance of a much more general class of dualities involving WZNW models on groups of rank $r>1$ and conformal Toda theory. We plan to return to this subject in the near future.

Acknowledgments: We are grateful to Thomas Creutzig, Vladimir Mitev, Ioannis Papadimitriou, Sylvain Ribault and Jörg Teschner for useful discussions and comments. The work of YH was supported by an JSPS Postdoctoral Fellowship for Research Abroad under contract number H18-143.

\section{A. The Lie superalgebras $\operatorname{osp}(1 \mid 2)$ and $\operatorname{sl}(1 \mid 2)$}

In this appendix we collect a few relevant details concerning the two Lie superalgebras that feature in the main text, namely the superalgebras $\operatorname{osp}(1 \mid 2)$ and $\operatorname{sl}(1 \mid 2)$.

\section{A.1 The Lie superalgebra $\operatorname{osp}(1 \mid 2)$}

The Lie superalgebra osp $(1 \mid 2)$ possesses three bosonic generators and two fermionic ones. We shall denote the former by $E^{ \pm}, H$ and use $F^{ \pm}$for fermionic generators. The relations between these elements are given by

$$
\begin{aligned}
{\left[H, E^{ \pm}\right] } & = \pm E^{ \pm}, & {\left[H, F^{ \pm}\right] } & = \pm \frac{1}{2} F^{ \pm}, & {\left[E^{+}, E^{-}\right] } & =2 H \\
{\left[E^{ \pm}, F^{\mp}\right] } & =-F^{ \pm}, & \left\{F^{+}, F^{-}\right\} & =\frac{1}{2} H, & \left\{F^{ \pm}, F^{ \pm}\right\} & = \pm \frac{1}{2} E^{ \pm} .
\end{aligned}
$$

Note that $E^{ \pm}$and $H$ generate a $\mathrm{sl}_{2}$ subalgebra within osp(1|2). It is easy to verify that the following matrices provide a 2|1-dimensional representation of $\operatorname{osp}(1 \mid 2)$ [39],

$$
\begin{array}{rlrl}
H & =\left(\begin{array}{ccc}
\frac{1}{2} & 0 & 0 \\
0 & -\frac{1}{2} & 0 \\
0 & 0 & 0
\end{array}\right), & E^{+}=\left(\begin{array}{ccc}
0 & 1 & 0 \\
0 & 0 & 0 \\
0 & 0 & 0
\end{array}\right), & E^{-}=\left(\begin{array}{lll}
0 & 0 & 0 \\
1 & 0 & 0 \\
0 & 0 & 0
\end{array}\right), \\
F^{+}=\left(\begin{array}{ccc}
0 & 0 & \frac{1}{2} \\
0 & 0 & 0 \\
0 & \frac{1}{2} & 0
\end{array}\right), & F^{-}=\left(\begin{array}{ccc}
0 & 0 & 0 \\
0 & 0 & -\frac{1}{2} \\
\frac{1}{2} & 0 & 0
\end{array}\right) .
\end{array}
$$


The Lie superalgebra osp $(1 \mid 2)$ possesses a non-degenerate invariant metric $\langle X, Y\rangle=\operatorname{str}(X Y)$ which is defined for any pair of elements $X, Y \in \operatorname{osp}(1 \mid 2)$ and using the supertrace in the 2|1-dimensional matrix representation,

$$
\langle H, H\rangle=\frac{1}{2},\left\langle E^{+}, E^{-}\right\rangle=\left\langle E^{-}, E^{+}\right\rangle=1,\left\langle F^{+}, F^{-}\right\rangle=-\left\langle F^{-}, F^{+}\right\rangle=\frac{1}{2} .
$$

The metric is needed e.g. to write down the action of a WZNW model on the Lie supergroup $\operatorname{OSP}(1 \mid 2)$.

\section{A.2 The Lie superalgebra $\operatorname{osp}(2 \mid 2)$}

The Lie superalgebra possesses four bosonic generators $E^{ \pm}, H$ and $Z$ along with four fermionic ones. The latter are denotes by $F^{ \pm}$and $\bar{F}^{ \pm}$. These eight generators obey the following set of non-trivial (anti-)commutation relations [39],

$$
\begin{aligned}
& {\left[H, E^{ \pm}\right]= \pm E^{ \pm}, \quad\left[H, F^{ \pm}\right]= \pm \frac{1}{2} F^{ \pm}, \quad\left[H, \bar{F}^{ \pm}\right]= \pm \frac{1}{2} \bar{F}^{ \pm},} \\
& {\left[Z, F^{ \pm}\right]=\frac{1}{2} F^{ \pm}, \quad\left[Z, \bar{F}^{ \pm}\right]=-\frac{1}{2} \bar{F}^{ \pm}, \quad\left[E^{+}, E^{-}\right]=2 H,} \\
& {\left[E^{ \pm}, F^{\mp}\right]=-F^{ \pm}, \quad\left[E^{ \pm}, \bar{F}^{\mp}\right]=\bar{F}^{ \pm}, \quad\left\{F^{ \pm}, \bar{F}^{\mp}\right\}=Z \mp H, \quad\left\{F^{ \pm}, \bar{F}^{ \pm}\right\}=E^{ \pm}}
\end{aligned}
$$

As in the case of $\operatorname{osp}(1 \mid 2)$ it is possible to find a matrix representation of osp(2|2) that is built out of 2|1-dimensional supermatrices,

$$
\begin{array}{ccc}
H=\left(\begin{array}{ccc}
\frac{1}{2} & 0 & 0 \\
0 & -\frac{1}{2} & 0 \\
0 & 0 & 0
\end{array}\right), & Z=\left(\begin{array}{ccc}
\frac{1}{2} & 0 & 0 \\
0 & \frac{1}{2} & 0 \\
0 & 0 & 1
\end{array}\right), & E^{+}=\left(\begin{array}{lll}
0 & 1 & 0 \\
0 & 0 & 0 \\
0 & 0 & 0
\end{array}\right), \\
F^{+}=\left(\begin{array}{lll}
0 & 0 & 0 \\
0 & 0 & 0 \\
0 & 1 & 0
\end{array}\right), & E^{-}=\left(\begin{array}{lll}
0 & 0 & 0 \\
1 & 0 & 0 \\
0 & 0 & 0
\end{array}\right),
\end{array}
$$

Using the prescription $\langle X, Y\rangle=\operatorname{str}(X Y)$ it is easy to find the following non-trivial bilinear form on $\operatorname{osp}(2 \mid 2)$,

$$
\begin{aligned}
& \langle H, H\rangle=\frac{1}{2}, \quad\langle Z, Z\rangle=-\frac{1}{2}, \quad\left\langle E^{+}, E^{-}\right\rangle=\left\langle E^{-}, E^{+}\right\rangle=1, \\
& \left\langle F^{+}, \bar{F}^{-}\right\rangle=\left\langle\bar{F}^{+}, F^{-}\right\rangle=1, \quad\left\langle\bar{F}^{-}, F^{+}\right\rangle=\left\langle F^{-}, \bar{F}^{+}\right\rangle=-1 .
\end{aligned}
$$

The form $\langle\cdot, \cdot\rangle$ defines a non-degenerate invariant metric on the Lie superalgebra osp $(2 \mid 2)$.

\section{B. Integral formulas}

In this appendix we list a few simple integral formulas that are used in some of the derivations we sketched in the main part of this note. 
The first formula concerns the overlap of two Bessel functions that is needed in section 3.1 on the minisuperspace theory. Utilizing the formula

$$
\begin{aligned}
& \int_{0}^{\infty} d x x^{\alpha-1} K_{\mu}(x) K_{\nu}(x)= \\
& =\frac{2^{\alpha-3}}{\Gamma(\alpha)} \Gamma\left(\frac{\alpha+\mu+\nu}{2}\right) \Gamma\left(\frac{\alpha+\mu-\nu}{2}\right) \Gamma\left(\frac{\alpha-\mu+\nu}{2}\right) \Gamma\left(\frac{\alpha-\mu-\nu}{2}\right),
\end{aligned}
$$

and

$$
\Gamma(i x) \Gamma(1-i x)=\frac{\pi}{i \sinh \pi x}, \quad \Gamma\left(\frac{1}{2}+i x\right) \Gamma\left(\frac{1}{2}-i x\right)=\frac{\pi}{\cosh \pi x},
$$

we obtain

$$
\begin{aligned}
& \int_{0}^{\infty} d x\left(K_{\frac{1+\epsilon}{2}+i p}(x) \pm K_{\frac{1+\epsilon}{2}-i p}(x)\right)\left(K_{\frac{1+\epsilon}{2}+i p^{\prime}}(x) \pm K_{\frac{1+\epsilon}{2}-i p^{\prime}}(x)\right) \\
& =\frac{1}{4 i}\left[\left(\frac{\pi}{\sinh \pi\left(\frac{p+p^{\prime}}{2}-i \epsilon\right)}-\frac{\pi}{\sinh \pi\left(\frac{p+p^{\prime}}{2}+i \epsilon\right)}\right) \frac{\pi}{\cosh \pi\left(\frac{p-p^{\prime}}{2}\right)}\right. \\
& \left.\quad \pm\left(\frac{\pi}{\sinh \pi\left(\frac{p-p^{\prime}}{2}-i \epsilon\right)}-\frac{\pi}{\sinh \pi\left(\frac{p-p^{\prime}}{2}+i \epsilon\right)}\right) \frac{\pi}{\cosh \pi\left(\frac{p+p^{\prime}}{2}\right)}\right] .
\end{aligned}
$$

If we take $\epsilon \rightarrow 0$, then the above quantity vanishes except for $p= \pm p^{\prime}$. Around these points we may use

$$
\frac{\pi}{\sinh \pi\left(\frac{p+p^{\prime}}{2}-i \epsilon\right)}-\frac{\pi}{\sinh \pi\left(\frac{p+p^{\prime}}{2}+i \epsilon\right)} \sim \frac{2 i \epsilon}{\left(\frac{p+p^{\prime}}{2}\right)^{2}+\epsilon^{2}} \rightarrow 4 \pi i \delta\left(p+p^{\prime}\right) .
$$

These results are exploited in our computation (3.11) of the particle 2-point correlator.

In passing from the 2-point function (3.16) in the $(x, \xi)$ basis to the $(\mu, \epsilon)$ basis, we make use of the following simple integrals,

$$
\begin{aligned}
& \frac{1}{2 \pi^{2}} \int \prod_{i=1,2}\left[\left|\mu_{i}\right|^{2 j_{i}+2} d^{2} x_{i} d \bar{\xi}_{i} d \xi_{i}\left(1-\epsilon_{i}\left|\mu_{i}\right| \xi_{i} \bar{\xi}_{i}\right) e^{\mu_{i} x_{i}-\bar{\mu}_{i} \bar{x}_{i}}\right] \delta^{2}\left(x_{1}-x_{2}\right)\left(\xi_{1}-\xi_{2}\right)\left(\bar{\xi}_{1}-\bar{\xi}_{2}\right)= \\
& \quad=-4\left|\mu_{2}\right|^{2} \epsilon_{2} \delta_{\epsilon_{1}, \epsilon_{2}} \delta^{2}\left(\mu_{1}+\mu_{2}\right)
\end{aligned}
$$

with $j_{1}+j_{2}+3 / 2=0$, and

$$
\begin{aligned}
& \frac{1}{2 \pi^{2}} \int \prod_{i=1,2}\left[\left|\mu_{i}\right|^{2 j_{i}+2} d^{2} x_{i} d \bar{\xi}_{i} d \xi_{i}\left(1-\epsilon_{i}\left|\mu_{i}\right| \xi_{i} \bar{\xi}_{i}\right) e^{\mu_{i} x_{i}-\bar{\mu}_{i} \bar{x}_{i}}\right]\left(1+\xi_{1} \xi_{2} \bar{\xi}_{1} \bar{\xi}_{2} \partial_{x_{1}} \partial_{\bar{x}_{1}}\right)\left|x_{1}-x_{2}\right|^{4 j_{1}+2}= \\
& \quad=4\left|\mu_{2}\right|^{2} \delta_{\epsilon_{1}, \epsilon_{2}} \delta^{2}\left(\mu_{1}+\mu_{2}\right) \pi \gamma\left(2 j_{2}+2\right)
\end{aligned}
$$

with $j_{1}-j_{2}=0$. Both formulas are straightforward to derive using only standard properties of Grassmann integrals. 
When computing three point functions, we use the function $D$ that is defined by the following integral formula

$$
D\left[\begin{array}{lll}
j_{1} & j_{2} & j_{3} \\
\mu_{1} & \mu_{2} & \mu_{3}
\end{array}\right]=\frac{1}{\pi^{3}} \int \prod_{i=1}^{3}\left[\left|\mu_{i}\right|^{2 j_{i}+2} d^{2} x_{i} e^{\mu_{i} x_{i}-\bar{\mu}_{i} \bar{x}_{i}}\right]\left|x_{12}\right|^{2 j_{12}}\left|x_{23}\right|^{2 j_{23}}\left|x_{31}\right|^{2 j_{31}},
$$

where $j_{12}=j_{1}+j_{2}-j_{3}$ and so on. The integrations may be performed explicitly and they lead to a rather bulky expression in terms of hypergeometric functions,

$$
\begin{aligned}
& D\left[\begin{array}{lll}
j_{1} & j_{2} & j_{3} \\
\mu_{1} & \mu_{2} & \mu_{3}
\end{array}\right]= \pi \delta^{(2)}\left(\mu_{1}+\mu_{2}+\mu_{3}\right)\left|\mu_{3}\right|^{-2 j_{1}-2 j_{2}-2}\left|\mu_{1}\right|^{2 j_{1}+2}\left|\mu_{2}\right|^{2 j_{2}+2} \times \\
& \times\left[\frac{\gamma\left(j_{31}+1\right) \gamma\left(j_{12}+1\right)}{\gamma(-j-1) \gamma\left(2 j_{1}+2\right)}{ }_{2} \mathcal{F}_{1}\left(j+2, j_{12}+1,2 j_{1}+2 ; 1+\frac{\mu_{2}}{\mu_{3}}\right)\right. \\
&\left.\quad+\left|1+\frac{\mu_{2}}{\mu_{3}}\right|^{-2\left(2 j_{1}+1\right)} \frac{\gamma\left(j_{23}+1\right)}{\gamma\left(-2 j_{1}\right)}{ }_{2} \mathcal{F}_{1}\left(-j_{31}, j_{23}+1,-2 j_{1} ; 1+\frac{\mu_{2}}{\mu_{3}}\right)\right] .
\end{aligned}
$$

Here we have used $j=j_{1}+j_{2}+j_{3}$ and

$$
{ }_{2} \mathcal{F}_{1}(a, b, c ; z)=F(a, b, c ; z) F(a, b, c ; \bar{z}) .
$$

\section{C. $\mathcal{N}=1$ super Liouville theory}

In order to carry out the computations of section 4 , we need rather extensive information on correlation functions in $\mathcal{N}=1$ Liouville field theory. For the convenience of the reader we collect all relevant formulas in this appendix. Most of the results are taken from [20].

In our conventions, the action of $\mathcal{N}=1$ super Liouville field theory takes the form

$$
S^{L}=\frac{1}{4 \pi} \int d^{2} z\left[\partial \varphi \bar{\partial} \varphi+\frac{Q}{4} \sqrt{g} R \varphi+\psi \bar{\partial} \psi+\bar{\psi} \partial \bar{\psi}\right]+i \mu_{L} b^{2} \int d^{2} z \psi \bar{\psi} e^{b \varphi},
$$

where $Q=b+1 / b$. For the relation with the OSP(1|2) WZNW model at level $k$ we set $b=1 / \sqrt{2 k-3}$ and fix the bulk cosmological constant to be $\mu_{L}=i /\left(2 \pi k b^{2}\right)$.

As all fermionic models, $\mathcal{N}=1$ Liouville theory possesses two sectors. Depending on the boundary conditions on fermions, these are denoted by NSNS (Neveu-Schwarz) and RR (Ramond) sectors. Primary fields in the NSNS sector can be thought of as exponentials $V_{\alpha}=e^{\alpha \varphi}$ in the bosonic field $\varphi$. Their conformal weight is given by $\Delta_{\alpha}^{L}=\alpha(Q-\alpha) / 2$. The 2-point function of these NSNS primary fields takes the form

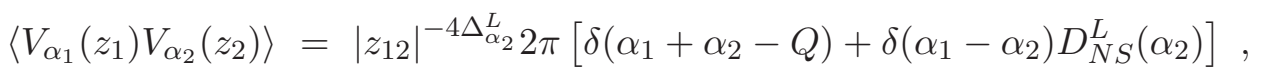

with

$$
D_{N S}^{L}(\alpha)=-\left(\mu_{L} \pi \gamma\left(\frac{b Q}{2}\right)\right)^{\frac{Q-2 \alpha}{b}} \frac{\Gamma\left(b\left(\alpha-\frac{Q}{2}\right)\right) \Gamma\left(\frac{1}{b}\left(\alpha-\frac{Q}{2}\right)\right)}{\Gamma\left(-b\left(\alpha-\frac{Q}{2}\right)\right) \Gamma\left(-\frac{1}{b}\left(\alpha-\frac{Q}{2}\right)\right)} .
$$

Here and throughout the main text we use $\gamma(x)=\Gamma(x) / \Gamma(1-x)$. Whereas the first term in eq. (C.10) is fixed by normalization, the second term involving $D_{N S}^{L}$ contains dynamical information on the phase shift of tachyonic modes upon reflection off the Liouville wall. 
The vertex operators that appear in our relation with the OSP (1|2) WZNW model, and in particular in eq. (4.2), are all in the RR sector. Before we can spell our properties of RR-fields we want to recall a few basic facts on spin fields which apply to $\mathcal{N}=1$ Liouville theory and free fermions alike. Chiral spin fields $\sigma^{ \pm}$and $\bar{\sigma}^{ \pm}$may be characterized by their operator product with the fermions,

$$
\psi(z) \sigma^{ \pm}(0) \sim \frac{\sigma^{\mp}(0)}{\sqrt{2} z^{\frac{1}{2}}}, \quad \quad \bar{\sigma}^{ \pm}(\bar{z}) \bar{\psi}(0) \sim \frac{i \bar{\sigma}^{\mp}(0)}{\sqrt{2} \bar{z}^{\frac{1}{2}}}
$$

As usual, we combine left- and right-moving spin fields into the non-chiral products $\sigma^{\epsilon \bar{\epsilon}}=$ $\sigma^{\epsilon} \bar{\sigma}^{\bar{\epsilon}}$. Their operator products are known to be given by

$$
\begin{array}{lll}
\sigma^{ \pm \pm}(z) \sigma^{ \pm \pm}(0) \sim \frac{1}{|z|^{\frac{1}{4}}}, & \sigma^{ \pm \mp}(z) \sigma^{ \pm \mp}(0) \sim \frac{i}{|z|^{\frac{1}{4}}} \\
\sigma^{ \pm \pm}(z) \sigma^{\mp \mp}(0) \sim-\frac{i}{2} \psi \bar{\psi}(0)|z|^{\frac{3}{4}}, & \sigma^{ \pm \mp}(z) \sigma^{\mp \pm}(0) \sim-\frac{1}{2} \psi \bar{\psi}(0)|z|^{\frac{3}{4}} .
\end{array}
$$

Only two special linear combinations of the spin fields play an important role for the theory. These are introduced as follows

$$
\Sigma^{+}=\frac{1}{\sqrt{2}}\left(\sigma^{++}-\sigma^{--}\right), \quad \Sigma^{-}=\frac{e^{-\pi i / 4}}{\sqrt{2}}\left(\sigma^{-+}-\sigma^{+-}\right) .
$$

In the case of the Ising model (free fermions), $\Sigma^{+}(=\sigma)$ is known as the order field while $\Sigma^{-}(=\mu)$ is referred to as the disorder field. From the operator products of spin fields we conclude easily,

$$
\Sigma^{+}(z) \Sigma^{+}(0) \sim \frac{1}{|z|^{\frac{1}{4}}}+\frac{i}{2} \psi \bar{\psi}|z|^{\frac{3}{4}}, \quad \Sigma^{-}(z) \Sigma^{-}(0) \sim \frac{1}{|z|^{\frac{1}{4}}}-\frac{i}{2} \psi \bar{\psi}|z|^{\frac{3}{4}} .
$$

$\mathcal{N}=1$ Liouville field theory contains a family of spin fields which is parametrized by their 'momentum' $\alpha$ in the $\varphi$ direction. We can think of these primary fields in the RR sector as products of a spin field and an exponential,

$$
\Theta_{\alpha}^{\epsilon \bar{\epsilon}}=\sigma^{\epsilon \bar{\epsilon}} e^{\alpha \varphi}, \quad \Sigma_{\alpha}^{ \pm}=\Sigma^{ \pm} e^{\alpha \varphi} .
$$

The 2-point functions of the vertex operators $\Sigma_{\alpha}^{\epsilon}$ possess the following form

$$
\left\langle\Sigma_{\alpha_{1}}^{ \pm}\left(z_{1}\right) \Sigma_{\alpha_{2}}^{ \pm}\left(z_{2}\right)\right\rangle=\left|z_{12}\right|^{-4 \Delta_{\alpha_{2}}^{L}-\frac{1}{4}} 2 \pi\left[\delta\left(\alpha_{1}+\alpha_{2}-Q\right) \mp \delta\left(\alpha_{1}-\alpha_{2}\right) D_{R}^{L}\left(\alpha_{2}\right)\right]
$$

with a reflection coefficient given by

$$
D_{R}^{L}(\alpha)=\left(\mu_{L} \pi \gamma\left(\frac{b Q}{2}\right)\right)^{\frac{Q-2 \alpha}{b}} \frac{\Gamma\left(\frac{1}{2}+b\left(\alpha-\frac{Q}{2}\right)\right) \Gamma\left(\frac{1}{2}+\frac{1}{b}\left(\alpha-\frac{Q}{2}\right)\right)}{\Gamma\left(\frac{1}{2}-b\left(\alpha-\frac{Q}{2}\right)\right) \Gamma\left(\frac{1}{2}-\frac{1}{b}\left(\alpha-\frac{Q}{2}\right)\right)} .
$$

In order to compute 3-point functions of $\operatorname{OSP}(1 \mid 2)$ model, we need 4-point functions of RR-fields involving a single degenerate field $\Theta_{-1 / 2 b}^{ \pm}$in $\mathcal{N}=1$ super Liouville theory. The 
states $|-1 / 2 b\rangle_{ \pm}=|1,2\rangle_{ \pm}$that correspond to the degenerate field are characterized by the relations,

$$
G_{0}|1,2\rangle_{ \pm}=\frac{i}{\sqrt{2}}\left[\frac{1}{b}+\frac{b}{2}\right]|1,2\rangle_{\mp}, \quad G_{-1}|1,2\rangle_{ \pm}+i \sqrt{2} b L_{-1}|1,2\rangle_{\mp}=0 .
$$

It follows from standard arguments that operator products involving at least one such degenerate field contain two terms only,

$$
-2 \Theta_{-1 / 2 b}^{ \pm \mp}\left(z_{1}\right) \Theta_{\alpha}^{\mp \pm}\left(z_{2}\right) \sim\left|z_{12}\right|^{\frac{\alpha}{b}+\frac{3}{4}} \psi \bar{\psi} V_{\alpha-1 / 2 b}\left(z_{2}\right)+C_{R,-}^{L}(\alpha)\left|z_{12}\right|^{\frac{1}{b}(Q-\alpha)-\frac{1}{4}} V_{\alpha+1 / 2 b}\left(z_{2}\right),
$$

where

$$
C_{R,-}^{L}(\alpha)=2 i D_{R}^{L}(\alpha) D_{N S}^{L}\left(Q-\alpha-\frac{1}{2 b}\right)=2 i b^{-2}\left(\mu_{L} \pi \gamma\left(\frac{b Q}{2}\right)\right)^{\frac{1}{b^{2}}} \gamma\left(\frac{1}{2}-\frac{\alpha}{b}\right) \gamma\left(\frac{\alpha}{b}-\frac{1}{2 b^{2}}\right) .
$$

The other operator product expansions we will need below can be obtained from the one we have provided by super conformal transformation along with the relations

$$
\Theta_{\alpha_{1}}^{\epsilon_{1}, \bar{\epsilon}_{1}}\left(z_{1}\right) \Theta_{\alpha_{2}}^{\epsilon_{2}, \bar{\epsilon}_{2}}\left(z_{2}\right) \sim-i \bar{\epsilon}_{1} \epsilon_{2} \Theta_{\alpha_{1}}^{-\epsilon_{1}, \bar{\epsilon}_{1}}\left(z_{1}\right) \Theta_{\alpha_{2}}^{-\epsilon_{2}, \bar{\epsilon}_{2}}\left(z_{2}\right) \sim-i \bar{\epsilon}_{1} \epsilon_{2} \Theta_{\alpha_{1}}^{\epsilon_{1},-\bar{\epsilon}_{1}}\left(z_{1}\right) \Theta_{\alpha_{2}}^{\epsilon_{2},-\bar{\epsilon}_{2}}\left(z_{2}\right) .
$$

Before we spell out a formula for the relevant 4-point functions, let us provide explicit expressions for the 3-point functions involving two RR fields. These were determined in $[40,41,20]$ and we shall simply quote their results along with all the necessary notations,

$$
\begin{aligned}
& \left\langle V_{\alpha_{1}}\left(z_{1}\right) \Theta_{\alpha_{2}}^{ \pm \pm}\left(z_{2}\right) \Theta_{\alpha_{3}}^{\mp \mp}\left(z_{3}\right)\right\rangle=\left|z_{12}\right|^{-2 \Delta_{12}^{L}}\left|z_{23}\right|^{-2 \Delta_{23}^{L}-\frac{1}{4}}\left|z_{31}\right|^{-2 \Delta_{31}^{L}} C_{R}^{L}\left(\alpha_{1} ; \alpha_{2}, \alpha_{3}\right), \\
& \left\langle V_{\alpha_{1}}\left(z_{1}\right) \Theta_{\alpha_{2}}^{ \pm \pm}\left(z_{2}\right) \Theta_{\alpha_{3}}^{ \pm \pm}\left(z_{3}\right)\right\rangle=\left|z_{12}\right|^{-2 \Delta_{12}^{L}}\left|z_{23}\right|^{-2 \Delta_{23}^{L}-\frac{1}{4}}\left|z_{31}\right|^{-2 \Delta_{31}^{L}} \tilde{C}_{R}^{L}\left(\alpha_{1} ; \alpha_{2}, \alpha_{3}\right),
\end{aligned}
$$

where $\Delta_{12}^{L}=\Delta_{\alpha_{1}}^{L}+\Delta_{\alpha_{2}}^{L}-\Delta_{\alpha_{3}}^{L}$ etc. Once more, other 3-point functions may be obtained with the help of the relations (C.13). The structure constants $C_{R}^{L}$ and $\tilde{C}_{R}^{L}$ are constructed from a special functions $\Upsilon$ as follows,

$$
\begin{aligned}
& C_{R}^{L}\left(\alpha_{1} ; \alpha_{2}, \alpha_{3}\right)=\left(\mu_{L} \pi \gamma\left(\frac{b Q}{2}\right) b^{1-b^{2}}\right)^{\frac{Q-\alpha}{b}} \frac{\Upsilon_{\mathrm{NS}}^{\prime}(0) \Upsilon_{\mathrm{NS}}\left(2 \alpha_{1}\right) \Upsilon_{\mathrm{R}}\left(2 \alpha_{2}\right) \Upsilon_{\mathrm{R}}\left(2 \alpha_{3}\right)}{\Upsilon_{\mathrm{R}}(\alpha-Q) \Upsilon_{\mathrm{R}}\left(\alpha_{23}\right) \Upsilon_{\mathrm{NS}}\left(\alpha_{12}\right) \Upsilon_{\mathrm{NS}}\left(\alpha_{31}\right)} \\
& \tilde{C}_{R}^{L}\left(\alpha_{1} ; \alpha_{2}, \alpha_{3}\right)=\left(\mu_{L} \pi \gamma\left(\frac{b Q}{2}\right) b^{1-b^{2}}\right)^{\frac{Q-\alpha}{b}} \frac{\Upsilon_{\mathrm{NS}}^{\prime}(0) \Upsilon_{\mathrm{NS}}\left(2 \alpha_{1}\right) \Upsilon_{\mathrm{R}}\left(2 \alpha_{2}\right) \Upsilon_{\mathrm{R}}\left(2 \alpha_{3}\right)}{\Upsilon_{\mathrm{NS}}(\alpha-Q) \Upsilon_{\mathrm{NS}}\left(\alpha_{23}\right) \Upsilon_{\mathrm{R}}\left(\alpha_{12}\right) \Upsilon_{\mathrm{R}}\left(\alpha_{31}\right)}
\end{aligned}
$$

where $\alpha_{12}=\alpha_{1}+\alpha_{2}-\alpha_{3}$ etc., $\alpha=\alpha_{1}+\alpha_{2}+\alpha_{3}$, and

$$
\Upsilon_{\mathrm{NS}}(x)=\Upsilon\left(\frac{x}{2}\right) \Upsilon\left(\frac{x+Q}{2}\right), \quad \Upsilon_{\mathrm{R}}(x)=\Upsilon\left(\frac{x+b}{2}\right) \Upsilon\left(\frac{x+b^{-1}}{2}\right) .
$$

The $\Upsilon$ function itself is closely related to Barnes double Gamma function. Instead of describing the precise connection, we simply display an integral representation

$$
\ln \Upsilon(x)=\int_{0}^{\infty} \frac{d t}{t}\left[e^{-2 t}\left(\frac{Q}{2}-x\right)^{2}-\frac{\sinh ^{2}\left(\frac{Q}{2}-x\right) t}{\sinh b t \sinh \frac{t}{b}}\right] .
$$


Note that the functions $\Upsilon_{\mathrm{NS}}$ and $\Upsilon_{\mathrm{R}}$ possess the following behavior under shifts of their argument,

$$
\begin{aligned}
\Upsilon_{\mathrm{NS}}(x+b) & =b^{-b x} \gamma\left(\frac{1}{2}+\frac{b x}{2}\right) \Upsilon_{\mathrm{R}}(x), & \Upsilon_{\mathrm{R}}(x+b) & =b^{1-b x} \gamma\left(\frac{b x}{2}\right) \Upsilon_{\mathrm{NS}}(x), \\
\Upsilon_{\mathrm{NS}}\left(x+\frac{1}{b}\right) & =b^{\frac{x}{b}} \gamma\left(\frac{1}{2}+\frac{x}{2 b}\right) \Upsilon_{\mathrm{R}}(x), & \Upsilon_{\mathrm{R}}\left(x+\frac{1}{b}\right) & =b^{-1+\frac{x}{b}} \gamma\left(\frac{x}{2 b}\right) \Upsilon_{\mathrm{NS}}(x)
\end{aligned}
$$

Let us finally turn to a discussion of the 4-point functions involving one degenerate field along with three primary fields from the RR sector. This quantity was computed in $[20]$ and it takes the form

$$
\begin{aligned}
& \left\langle\Sigma_{-1 / 2 b}^{\epsilon_{0}}\left(z_{0}\right) \Sigma_{\alpha_{1}}^{\epsilon_{1}}\left(z_{1}\right) \Sigma_{\alpha_{2}}^{\epsilon_{2}}\left(z_{2}\right) \Sigma_{\alpha_{3}}^{\epsilon_{3}}\left(z_{3}\right)\right\rangle=\left|z_{03}\right|^{-4 \Delta_{-1 / 2 b}^{L}-\frac{1}{4}} \times \\
& \quad \times\left|z_{12}\right|^{-2 \Delta_{12}^{L}-\frac{1}{4}-2 \Delta_{-1 / 2 b}^{L}}\left|z_{23}\right|^{-2 \Delta_{23}^{L}+2 \Delta_{-1 / 2 b}^{L}}\left|z_{31}\right|^{-2 \Delta_{31}^{L}+2 \Delta_{-1 / 2 b}^{L}} \mathcal{H}^{\epsilon_{0} \epsilon_{1} \epsilon_{2} \epsilon_{3}}(z)
\end{aligned}
$$

where $\mathcal{H}^{\epsilon_{0} \epsilon_{1} \epsilon_{2} \epsilon_{3}}(z)$ is a function of the cross ratio $z=\left(z_{01} z_{23}\right) /\left(z_{03} z_{21}\right)$. We need some preparation before we can specify the functions $\mathcal{H}$. They are built from yet another set of auxiliary functions which depend on $\alpha_{i}=Q / 2+i p_{i}$ according to

$$
\begin{aligned}
& \mathcal{G}_{0}\left(p_{1}, p_{2}, p_{3} ; z\right)=-\frac{1}{2}(z(1-z))^{\frac{5}{8}} F\left(\frac{5}{4}, \frac{3}{4}, \frac{3}{2}, z\right)\left(G\left(p_{1}, p_{2}, p_{3} ; z\right)-G\left(p_{1},-p_{2},-p_{3} ; z\right)\right) \\
& \mathcal{G}_{1}\left(p_{1}, p_{2}, p_{3} ; z\right)=(z(1-z))^{\frac{1}{8}} F\left(\frac{1}{4},-\frac{1}{4}, \frac{1}{2}, z\right)\left(G\left(p_{1}, p_{2}, p_{3} ; z\right)+G\left(p_{1},-p_{2},-p_{3} ; z\right)\right)
\end{aligned}
$$

where

$$
G\left(p_{1}, p_{2}, p_{3} ; z\right)=z^{\frac{1}{4 b^{2}}+\frac{i p_{1}}{2 b}}(1-z)^{\frac{1}{4 b^{2}}+\frac{i p_{2}}{2 b}}\left(\frac{\frac{1}{4}+\frac{i}{2 b} p_{31}}{\frac{1}{2}+\frac{i}{b} p_{1}}\right) F\left(\frac{3}{4}+\frac{i}{2 b} p, \frac{1}{4}+\frac{i}{2 b} p_{12}, \frac{3}{2}+\frac{i}{b} p_{1} ; z\right)
$$

and $p_{12}=p_{1}+p_{2}-p_{3}$ etc., $p=p_{1}+p_{2}+p_{3}$. One may show that $\mathcal{H}$ and $\mathcal{G}_{a}$ obey the same linear differential equations. Hence, we will be able to construct $\mathcal{H}$ from $\mathcal{G}_{0}, \mathcal{G}_{1}$ and

$$
\mathcal{G}_{2}\left(p_{1}, p_{2}, p_{3} ; z\right)=\mathcal{G}_{0}\left(-p_{1}, p_{2},-p_{3} ; z\right), \quad \mathcal{G}_{3}\left(p_{1}, p_{2}, p_{3} ; z\right)=\mathcal{G}_{1}\left(-p_{1}, p_{2},-p_{3} ; z\right)
$$

Combinations of these four functions $\mathcal{G}_{a}$ with trivial monodromies around $z=0$ and $z=1$ are given by (see also [20])

$$
\begin{aligned}
H_{1}^{ \pm}\left(p_{1}, p_{2}, p_{3} ; z\right) & =\left( \pm \mathcal{G}_{0} \overline{\mathcal{G}}_{0}+\mathcal{G}_{1} \overline{\mathcal{G}}_{1}\right)+\gamma\left(\frac{1}{2}+\frac{i}{b} p_{1}\right)^{2} \gamma\left(\frac{1}{4}+\frac{i}{2 b} p_{23}\right) \times \\
& \times \gamma\left(\frac{1}{4}-\frac{i}{2 b} p\right) \gamma\left(\frac{3}{4}-\frac{i}{2 b} p_{31}\right) \gamma\left(\frac{3}{4}-\frac{i}{2 b} p_{12}\right)\left(\mathcal{G}_{2} \overline{\mathcal{G}}_{2} \pm \mathcal{G}_{3} \overline{\mathcal{G}}_{3}\right), \\
H_{2}^{ \pm}\left(p_{1}, p_{2}, p_{3} ; z\right) & =\left(\mathcal{G}_{0} \overline{\mathcal{G}}_{1} \pm \mathcal{G}_{1} \overline{\mathcal{G}}_{0}\right)+\gamma\left(\frac{1}{2}+\frac{i}{b} p_{1}\right)^{2} \gamma\left(\frac{1}{4}+\frac{i}{2 b} p_{23}\right) \times \\
& \times \gamma\left(\frac{1}{4}-\frac{i}{2 b} p\right) \gamma\left(\frac{3}{4}-\frac{i}{2 b} p_{13}\right) \gamma\left(\frac{3}{4}-\frac{i}{2 b} p_{12}\right)\left( \pm \mathcal{G}_{2} \overline{\mathcal{G}}_{3}+\mathcal{G}_{3} \overline{\mathcal{G}}_{2}\right)
\end{aligned}
$$

where $\overline{\mathcal{G}}_{i}\left(p_{1}, p_{2}, p_{3} ; z\right)=\mathcal{G}_{i}\left(p_{1}, p_{2}, p_{3} ; \bar{z}\right)$. Both $H_{1}^{+}$and $H_{2}^{-}$have previously appeared in [20] where they were also shown to be invariant under the crossing symmetry transformation $z \mapsto 1-z$ (note that $H_{2}^{-}$flips its sign). Under the action of the same crossing symmetry transformation, our functions $H_{1}^{-}$and $H_{2}^{+}$are mapped onto each other. We finally combine 
the functions $H_{1}$ and $H_{2}$ into $\mathcal{H}$, in a way that is determined by the desired operator product expansions,

$$
\begin{aligned}
& 2 \mathcal{H}^{ \pm \pm \pm \pm}(z)=\mp C_{R}^{L}\left(\tilde{\alpha}_{1}, \alpha_{2}, \alpha_{3}\right) H_{1}^{+}\left(p_{1}, p_{2}, p_{3} ; z\right)+\tilde{C}_{R}^{L}\left(\tilde{\alpha}_{1}, \alpha_{2}, \alpha_{3}\right) H_{1}^{+}\left(p_{1}, p_{2},-p_{3} ; z\right), \\
& 2 \mathcal{H}^{ \pm \pm \mp \mp}(z)= \pm C_{R}^{L}\left(\tilde{\alpha}_{1}, \alpha_{2}, \alpha_{3}\right) H_{1}^{-}\left(p_{1}, p_{2}, p_{3} ; z\right)+\tilde{C}_{R}^{L}\left(\tilde{\alpha}_{1}, \alpha_{2}, \alpha_{3}\right) H_{1}^{-}\left(p_{1}, p_{2},-p_{3} ; z\right), \\
& 2 \mathcal{H}^{ \pm \mp \pm \mp}(z)=-C_{R}^{L}\left(\tilde{\alpha}_{1}, \alpha_{2}, \alpha_{3}\right) H_{2}^{+}\left(p_{1}, p_{2}, p_{3} ; z\right) \pm \tilde{C}_{R}^{L}\left(\tilde{\alpha}_{1}, \alpha_{2}, \alpha_{3}\right) H_{2}^{+}\left(p_{1}, p_{2},-p_{3} ; z\right), \\
& 2 \mathcal{H}^{ \pm \mp \mp \pm}(z)=\mp i C_{R}^{L}\left(\tilde{\alpha}_{1}, \alpha_{2}, \alpha_{3}\right) H_{2}^{-}\left(p_{1}, p_{2}, p_{3} ; z\right)+i \tilde{C}_{R}^{L}\left(\tilde{\alpha}_{1}, \alpha_{2}, \alpha_{3}\right) H_{2}^{-}\left(p_{1}, p_{2},-p_{3} ; z\right),
\end{aligned}
$$

where the first argument of $C_{R}^{L}$ and $\tilde{C}_{R}^{L}$ is shifted by $1 / 2 b$, i.e. we have set $\tilde{\alpha}=\alpha-1 / 2 b$. This concludes our description of the 4-point functions.

\section{References}

[1] R. R. Metsaev and A. A. Tseytlin, Type IIB superstring action in $A d S_{5} \times S^{5}$ background, Nucl. Phys. B 533 (1998) 109 [hep-th/9805028].

[2] K. B. Efetov, Supersymmetry and theory of disordered metals, Adv. Phys. 32 (1983) 53.

[3] D. Bernard, (Perturbed) conformal field theory applied to 2D disordered systems: An introduction, hep-th/9509137.

[4] V. Schomerus and H. Saleur, The GL(1|1) WZW model: From supergeometry to logarithmic CFT, Nucl. Phys. B 734 (2006) 221 [hep-th/0510032].

[5] H. Dorn and H. J. Otto, Two and three point functions in Liouville theory, Nucl. Phys. B 429 (1994) 375 [hep-th/9403141].

[6] A. B. Zamolodchikov and A. B. Zamolodchikov, Structure constants and conformal bootstrap in Liouville field theory, Nucl. Phys. B 477 (1996) 577 [hep-th/9506136].

[7] B. Ponsot and J. Teschner, Liouville bootstrap via harmonic analysis on a noncompact quantum group, hep-th/9911110.

[8] J. Teschner, Liouville theory revisited, Class. and Quant. Grav. 18 (2001) R153 [hep-th/0104158].

[9] J. Teschner, A lecture on the Liouville vertex operators, Int. J. Mod. Phys. A 19S2 (2004) $436[\mathrm{hep}-\mathrm{th} / 0303150]$.

[10] J. Teschner, On structure constants and fusion rules in the $S L(2, \mathbb{C}) / S U(2) W Z N W$ model, Nucl. Phys. B 546 (1999) 390 [hep-th/9712256].

[11] J. Teschner, Operator product expansion and factorization in the $\mathrm{H}_{3}^{+}$WZNW model, Nucl. Phys. B 571 (2000) 555 [hep-th/9906215].

[12] J. Teschner, Crossing symmetry in the $H_{3}^{+}$WZNW model, Phys. Lett. B 521 (2001) 127 [hep-th/0108121].

[13] S. Ribault and J. Teschner, $H_{3}^{+}$WZNW correlators from Liouville theory, J. High Energy Phys. 06 (2005) 014 [hep-th/0502048].

[14] A. V. Stoyanovsky, A relation between the Knizhnik-Zamolodchikov and Belavin-Polyakov-Zamolodchikov systems of partial differential equations, math-ph/0012013.

[15] E. K. Sklyanin, Separation of variables in the Gaudin model, J. Sov. Math. 47 (1989) 2473 [Zap. Nauchn. Semin. 164 (1987) 151].

[16] Y. Hikida and V. Schomerus, $H_{3}^{+}$WZNW model from Liouville field theory, J. High Energy Phys. 10 (2007) 064 [arXiv:0706.1030].

[17] P. Bouwknegt and K. Schoutens, W symmetry in conformal field theory, Phys. Rept. 223 (1993) 183 [hep-th/9210010].

[18] T. Quella and V. Schomerus, Free fermion resolution of supergroup WZNW models, J. High Energy Phys. 09 (2007) 085 [arXiv:0706.0744]. 
[19] M. Bershadsky and H. Ooguri, Hidden $\operatorname{OSp}(N, 2)$ symmetries in superconformal field theory, Phys. Lett. B 229 (1989) 374.

[20] T. Fukuda and K. Hosomichi, Super Liouville theory with boundary, Nucl. Phys. B 635 (2002) 215 [hep-th/0202032].

[21] H. Saleur and B. Wehefritz Kaufmann, Integrable quantum field theories with supergroup symmetries: The OSP(1/2) case, Nucl. Phys. B 663 (2003) 443 [hep-th/0302144].

$[22]$ F. H. L. Essler, H. Frahm and H. Saleur, Continuum limit of the integrable sl(2/1) $3-\overline{3}$ superspin chain, Nucl. Phys. B 712 (2005) 513 [cond-mat/0501197].

[23] D. Bernard and A. LeClair, Spin-charge separation and the spin quantum Hall effect, Phys. Rev. B 64 (2001) 045306 [cond-mat/0003075].

[24] M. J. Bhaseen, J. S. Caux, I. I. Kogan and A. M. Tsvelik, Disordered Dirac fermions: The marriage of three different approaches, Nucl. Phys. B 618 (2001) 465 [cond-mat/0012240].

[25] A. LeClair, The gl(1|1) super-current algebra: The role of twist and logarithmic fields, arXiv:0710.2906.

[26] P. Di Francesco, P. Mathieu and D. Sénéchal, Conformal field theory, Springer (1997).

[27] A. A. Belavin, A. M. Polyakov and A. B. Zamolodchikov, Infinite conformal symmetry in two-dimensional quantum field theory, Nucl. Phys. B 241 (1984) 333.

[28] A. Y. Alekseev and V. Schomerus, D-branes in the WZW model, Phys. Rev. D 60 (1999) 061901 [hep-th/9812193].

[29] T. Creutzig, T. Quella and V. Schomerus, Branes in the GL(1|1) WZNW-Model, arXiv:0708.0583.

[30] C. Ahn, C. Rim and M. Stanishkov, Exact one-point function of $\mathcal{N}=1$ super-Liouville theory with boundary, Nucl. Phys. B 636 (2002) 497 [hep-th/0202043].

[31] K. Hosomichi and S. Ribault, Solution of the $\mathrm{H}_{3}^{+}$model on a disc, J. High Energy Phys. 01 (2007) 057 [hep-th/0610117].

[32] V. Fateev and S. Ribault, Boundary action of the $H_{3}^{+}$model, arXiv:0710.2093.

[33] C. Ahn, C. Kim, C. Rim and M. Stanishkov, Duality in $\mathcal{N}=2$ super-Liouville theory, Phys. Rev. D 69 (2004) 106011 [hep-th/0210208].

[34] K. Hosomichi, $\mathcal{N}=2$ Liouville theory with boundary, J. High Energy Phys. 12 (2006) 061 [hep-th/0408172].

[35] H. Saleur and V. Schomerus, On the $S U(2 \mid 1)$ WZNW model and its statistical mechanics applications, Nucl. Phys. B 775 (2007) 312 [hep-th/0611147].

[36] Z. Maassarani and D. Serban, Non-unitary conformal field theory and logarithmic operators for disordered systems, Nucl. Phys. B 489 (1997) 603 [hep-th/9605062].

[37] A. W. W. Ludwig, A free field representation of the Osp(2|2) current algebra at level $k=-2$, and Dirac fermions in a random SU(2) gauge potential, cond-mat/0012189.

[38] P. Forgacs, A. Wipf, J. Balog, L. Feher and L. O'Raifeartaigh, Liouville and Toda theories as conformally reduced WZNW theories, Phys. Lett. B 227 (1989) 214.

[39] L. Frappat, P. Sorba and A. Sciarrino, Dictionary on Lie superalgebras, hep-th/9607161.

[40] R. C. Rashkov and M. Stanishkov, Three-point correlation functions in $\mathcal{N}=1$ super Liouville theory, Phys. Lett. B 380 (1996) 49 [hep-th/9602148].

[41] R. H. Poghosian, Structure constants in the $\mathcal{N}=1$ super-Liouville field theory, Nucl. Phys. B 496 (1997) 451 [hep-th/9607120]. 